\title{
Strong convergence theorems for a generalized mixed equilibrium problem and variational inequality problems
}

\section{Jae Ug Jeong*}

"Correspondence:

jujeong@deu.ac.kr

Department of Mathematics,

Dongeui University, Busan, 614-714,

South Korea

\begin{abstract}
In this paper, a new iterative scheme based on the extragradient-like method for finding a common element of the set of common fixed points of a finite family of nonexpansive mappings, the set of solutions of variational inequalities for a strongly positive linear bounded operator and the set of solutions of a mixed equilibrium problem is proposed. A strong convergence theorem for this iterative scheme in Hilbert spaces is established. Our results extend recent results announced by many others.
\end{abstract}

MSC: 49J30; 49J40; 47J25; 47H09

Keywords: fixed point; inverse strongly monotone mapping; variational inequality; equilibrium problem

\section{Introduction}

Let $H$ be a real Hilbert space with the inner product $\langle\cdot, \cdot\rangle$ and the norm $\|\cdot\|$. Let $C$ be a nonempty closed convex subset of $H$. Recall that a mapping $T: C \rightarrow C$ is said to be nonexpansive if

$$
\|T x-T y\| \leq\|x-y\|, \quad \forall x, y \in C .
$$

We denote by $F(T)$ the set of fixed points of $T$. Let $P_{C}$ be the projection of $H$ onto the convex subset $C$. Moreover, we also denote by $\mathbb{R}$ the set of all real numbers.

Peng and Yao [1] considered the generalized mixed equilibrium problem of finding $x \in C$ such that

$$
\Theta(x, y)+\varphi(y)-\varphi(x)+\langle F x, y-x\rangle \geq 0, \quad \forall y \in C,
$$

where $F: C \rightarrow H$ is a nonlinear mapping and $\varphi: C \rightarrow \mathbb{R}$ is a function and $\Theta: C \times C \rightarrow \mathbb{R}$ is a bifunction. The set of solutions of problem (1.1) is denoted by GMEP.

In the case of $F=0$, problem (1.1) reduces to the mixed equilibrium problem of finding $x \in C$ such that

$$
\Theta(x, y)+\varphi(y)-\varphi(x) \geq 0, \quad \forall y \in C,
$$

which was considered by Ceng and Yao [2]. GMEP is denoted by MEP.

2 2013 Jeong: licensee Springer. This is an Open Access article distributed under the terms of the Creative Commons Attribution License (http://creativecommons.org/licenses/by/2.0), which permits unrestricted use, distribution, and reproduction in any medium, provided the original work is properly cited. 
In the case of $\varphi=0$, problem (1.1) reduces to the generalized equilibrium problem of finding $x \in C$ such that

$$
\Theta(x, y)+\langle F x, y-x\rangle \geq 0, \quad \forall y \in C
$$

which was studied by Takahashi and Takahashi [3] and many others, for example, [4-10].

In the case of $\varphi=0$ and $F=0$, problem (1.1) reduces to the equilibrium problem of finding $x \in C$ such that

$$
\Theta(x, y) \geq 0, \quad \forall y \in C
$$

The set of solutions of (1.2) is denoted by $\operatorname{EP}(\Theta)$.

In the case $\Theta=0, \varphi=0$ and $F=A$, problem (1.1) reduces to the classical variational inequality problem of finding $x \in C$ such that

$$
\langle A x, y-x\rangle \geq 0, \quad \forall y \in C
$$

The set of solutions of problem (1.3) is denoted by $\operatorname{VI}(A, C)$.

Problem (1.1) is very general in the sense that it includes, as special cases, optimization problems, variational inequalities, minimax problems, the Nash equilibrium problem in noncooperative games and others; see, for instance, [2, 3, 11]. Peng and Yao [1] considered iterative methods for finding a common element of the set of solutions of problem (1.1), the set of solutions of problem (1.2) and the set of fixed points of a nonexpansive mapping.

Let $G_{1}, G_{2}: C \times C \rightarrow \mathbb{R}$ be two bifunctions and let $B_{1}, B_{2}: C \rightarrow H$ be two nonlinear mappings. We consider the generalized equilibrium problem $(\bar{x}, \bar{y}) \in C \times C$ such that

$$
\begin{cases}G_{1}(\bar{x}, x)+\left\langle B_{1} \bar{y}, x-\bar{x}\right\rangle+\frac{1}{\mu_{1}}\langle\bar{x}-\bar{y}, x-\bar{x}\rangle \geq 0, & \forall x \in C, \\ G_{2}(\bar{y}, y)+\left\langle B_{2} \bar{x}, y-\bar{y}\right\rangle+\frac{1}{\mu_{2}}\langle\bar{y}-\bar{x}, y-\bar{y}\rangle \geq 0, \quad \forall y \in C,\end{cases}
$$

where $\mu_{1}>0$ and $\mu_{2}>0$ are two constants.

In the case $G_{1}=G_{2}=0$, problem (1.4) reduces to the general system of variational inequalities of finding $(\bar{x}, \bar{y}) \in C \times C$ such that

$$
\begin{cases}\left\langle\mu_{1} B_{1} \bar{y}+\bar{x}-\bar{y}, x-\bar{x}\right\rangle \geq 0, & \forall x \in C \\ \left\langle\mu_{2} B_{2} \bar{x}+\bar{y}-\bar{x}, y-\bar{y}\right\rangle \geq 0, & \forall y \in C\end{cases}
$$

where $\mu_{1}>0$ and $\mu_{2}>0$ are two constants, which was considered by Ceng, Wang and Yao [12]. In particular, if $B_{1}=B_{2}=A$, then problem (1.5) reduces to the system of variational inequalities of finding $(\bar{x}, \bar{y}) \in C \times C$ such that

$$
\begin{cases}\left\langle\mu_{1} A \bar{y}+\bar{x}-\bar{y}, x-\bar{x}\right\rangle \geq 0, & \forall x \in C, \\ \left\langle\mu_{2} A \bar{x}+\bar{y}-\bar{x}, y-\bar{y}\right\rangle \geq 0, \quad \forall y \in C,\end{cases}
$$

which was studied by Verma [13]. 
If $\bar{x}=\bar{y}$ in (1.6), then (1.6) reduces to the classical variational inequality (1.3). Further, problem (1.6) is equivalent to the following projection formulas:

$$
\left\{\begin{array}{l}
\bar{x}=P_{C}\left(I-\mu_{1} A\right) \bar{y}, \\
\bar{y}=P_{C}\left(I-\mu_{2} A\right) \bar{x} .
\end{array}\right.
$$

Recently, Ceng et al. [12] introduced and studied a relaxed extragradient method for finding solutions of problem (1.5).

Let $\left\{T_{i}\right\}$ be an infinite family of nonexpansive mappings of $C$ into itself and $\left\{\lambda_{n 1}\right\},\left\{\lambda_{n 2}\right\}$, $\ldots,\left\{\lambda_{n N}\right\}$ be real sequences such that $\lambda_{n 1}, \lambda_{n 2}, \ldots, \lambda_{n N} \in(0,1]$ for every $n \in N$. For any $n \geq$ 1, we define a mapping $W_{n}$ of $C$ into itself as follows:

$$
\begin{aligned}
& U_{n 0}=I, \\
& U_{n 1}=\lambda_{n 1} T_{1} U_{n 0}+\left(1-\lambda_{n 1}\right) I, \\
& U_{n 2}=\lambda_{n 2} T_{2} U_{n 1}+\left(1-\lambda_{n 2}\right) I, \\
& \quad \vdots \\
& U_{n, N-1}=\lambda_{n, N-1} T_{N-1} U_{n, N-2}+\left(1-\lambda_{n, N-1}\right) I, \\
& W_{n}=U_{n, N}=\lambda_{n, N} T_{N} U_{n, N-1}+\left(1-\lambda_{n, N}\right) I .
\end{aligned}
$$

Such a mapping $W_{n}$ is called the $W$-mapping generated by $T_{1}, T_{2}, \ldots, T_{N}$ and $\left\{\lambda_{n 1}\right\},\left\{\lambda_{n 2}\right\}$, $\ldots,\left\{\lambda_{n N}\right\}$. Nonexpansivity of each $T_{i}$ ensures the nonexpansivity of $W_{n}$. Moreover, in [1], it is shown that $F\left(W_{n}\right)=\bigcap_{i=1}^{N} F\left(T_{i}\right)$.

Throughout this article, let us assume that a bifunction $\Theta: C \times C \rightarrow \mathbb{R}$ and a convex function $\varphi: C \rightarrow \mathbb{R}$ satisfy the following conditions:

(H1) $\Theta(x, x)=0$ for all $x \in C$;

(H2) $\Theta$ is monotone, i.e., $\Theta(x, y)+\Theta(y, x) \leq 0$ for all $x, y \in C$;

(H3) for each $y \in C, x \mapsto \Theta(x, y)$ is weakly upper semicontinuous;

(H4) for each $x \in C, y \mapsto \Theta(x, y)$ is convex and lower semicontinuous;

(A1) for each $x \in H$ and $r>0$, there exists a bounded subset $D_{x} \subset C$ and $y_{x} \in C$ such that for any $z \in C \backslash D_{x}$,

$$
\Theta\left(z, y_{x}\right)+\varphi\left(y_{x}\right)-\varphi(z)+\frac{1}{r}\left\langle y_{x}-z, z-x\right\rangle<0
$$

(A2) $C$ is a bounded set.

Recently, Qin et al. [8] studied the problem of finding a common element of the set of common fixed points of a finite family of nonexpansive mappings, the set of solutions of variational inequalities for a relaxed cocoercive mapping and the set of solutions of an equilibrium problem. More precisely, they proved the following theorem.

Theorem 1.1 Let $C$ be a nonempty closed convex subset of a real Hilbert space H. Let $\Theta$ be a bifunction from $C \times C$ to $\mathbb{R}$ which satisfies $(H 1)-(H 4)$. Let $T_{1}, T_{2}, \ldots, T_{N}$ be a finite family of nonexpansive mappings of $C$ into $H$ and let $B$ be a $\mu$-Lipschitz, relaxed $(u, v)$-cocoercive mapping ofC into H such that $F=\bigcap_{i=1}^{N} F\left(T_{i}\right) \cap \mathrm{EP}(\Theta) \cap \mathrm{VI}(A, C) \neq \phi$. Letf be a contraction 
of H into itself with a coefficient $\alpha(0<\alpha<1)$ and let $A$ be a strongly positive linear bounded operator with a coefficient $\bar{\gamma}>0$ such that $\|A\| \leq 1$. Assume that $0<\gamma<\frac{\bar{\gamma}}{\alpha}$. Let $\left\{x_{n}\right\}$ and $\left\{y_{n}\right\}$ be sequences generated by $x_{1} \in H$ and

$$
\left\{\begin{array}{l}
\Theta\left(y_{n}, \eta\right)+\frac{1}{r_{n}}\left\langle\eta-y_{n}, y_{n}-x_{n}\right\rangle \geq 0, \quad \forall \eta \in C, \\
x_{n+1}=\alpha_{n} \gamma f\left(W_{n} x_{n}\right)+\left(1-\alpha_{n} A\right) W_{n} P_{C}\left(I-s_{n} B\right) y_{n}, \quad n \geq 1,
\end{array}\right.
$$

where $\alpha_{n} \in(0,1]$ and $\left\{r_{n}\right\},\left\{s_{n}\right\} \subset[0, \infty)$ satisfy

(i) $\lim _{n \rightarrow \infty} \alpha_{n}=0$ and $\sum_{n=1}^{\infty} \alpha_{n}=\infty$;

(ii) $\sum_{n=1}^{\infty}\left|\alpha_{n+1}-\alpha_{n}\right|<\infty, \sum_{n=1}^{\infty}\left|r_{n+1}-r_{n}\right|<\infty$ and $\sum_{n=1}^{\infty}\left|s_{n+1}-s_{n}\right|<\infty$;

(iii) $\liminf _{n \rightarrow \infty} r_{n}>0$;

(iv) $\left\{s_{n}\right\} \subset[a, b]$ for some $a, b$ with $0 \leq a \leq b \leq \frac{2\left(v-u \mu^{2}\right)}{\mu^{2}}, v \geq u \mu^{2}$;

(v) $\sum_{n=0}^{\infty}\left|\lambda_{n, i}-\lambda_{n-1, i}\right|<\infty$ for all $i=1,2, \ldots, N$.

Then, both $\left\{x_{n}\right\}$ and $\left\{y_{n}\right\}$ converge strongly to $q \in F$, where $q=P_{F}(\gamma f+(I-A))(q)$, which solves the following variational inequality:

$$
\langle\gamma f(q)-A q, p-q\rangle \leq 0, \quad \forall p \in F .
$$

In this paper, motivated by Takahashi and Takahashi [3], Ceng, Wang and Yao [12], Peng and Yao [1] and Qin, Shang and Su [8], we introduce the general iterative scheme for finding a common element of the set of common fixed points of a finite family of nonexpansive mappings, the set of solutions of the generalized mixed equilibrium problem (1.1) and the set of solutions of the generalized equilibrium problem (1.4), which solves the variational inequality

$$
\left\langle(A-\gamma f) x^{*}, x-x^{*}\right| \geq 0, \quad \forall x \in \mathfrak{F},
$$

where $\mathfrak{F}=\bigcap_{i=1}^{N} F\left(T_{i}\right) \cap$ GMEP $\cap \Omega$ and $\Omega$ is the set of solutions of the generalized equilibrium problem (1.4). The results obtained in this paper improve and extend the recent results announced by Qin et al. [8], Chen et al. [14], Combetters and Hirstoaga [15], Iiduka and Takahashi [16], Marino and Xu [17], Takahashi and Takahashi [18], Wittmann [19] and many others.

\section{Preliminaries}

Let $C$ be a nonempty closed convex subset of a real Hilbert space $H$. For every point $x \in H$, there exists a unique nearest point of $C$, denoted by $P_{C} x$, such that $\left\|x-P_{C} x\right\| \leq\|x-y\|$ for all $y \in C$. Such a $P_{C}$ is called the metric projection of $H$ onto $C$. We know that $P_{C}$ is a firmly nonexpansive mapping of $H$ onto $C$, i.e.,

$$
\left\langle x-y, P_{C} x-P_{C} y\right\rangle \geq\left\|P_{C} x-P_{C} y\right\|^{2}, \quad \forall x, y \in H .
$$

Further, for any $x \in H$ and $z \in C, z=P_{C} x$ if and only if

$$
\langle x-z, z-y\rangle \geq 0, \quad \forall y \in C .
$$


It is also known that $H$ satisfies Opial's condition [20] if for each sequence $\left\{x_{n}\right\}_{n=1}^{\infty}$ in $H$ which converges weakly to a point $x \in H$, we have

$$
\liminf _{n \rightarrow \infty}\left\|x_{n}-x\right\|<\liminf _{n \rightarrow \infty}\left\|x_{n}-y\right\|, \quad \forall y \in H, y \neq x
$$

Moreover, we assume that $A$ is a bounded strongly positive operator on $H$ with a constant $\bar{\gamma}$, that is, there exists $\bar{\gamma}>0$ such that

$$
\langle A x, x\rangle \geq \bar{\gamma}\|x\|^{2}, \quad \forall x \in H
$$

A mapping $B: C \rightarrow H$ is called $\beta$-inverse strongly monotone if there exists $\beta>0$ such that

$$
\langle x-y, B x-B y\rangle \geq \beta\|B x-B y\|^{2}, \quad \forall x, y \in C .
$$

It is obvious that any inverse strongly monotone mapping is Lipschitz continuous.

In order to prove our main results in the next section, we need the following lemmas and proposition.

Lemma 2.1 [2] Let $C$ be a nonempty closed convex subset of $H$. Let $\Theta: C \times C \rightarrow \mathbb{R}$ be a bifunction satisfying conditions (H1)-(H4) and let $\varphi: C \rightarrow \mathbb{R}$ be a lower semicontinuous and convex function. For $r>0$ and $x \in H$, define a mapping

$$
T_{r}^{(\Theta, \varphi)}(x)=\left\{z \in C: \Theta(z, y)+\varphi(y)-\varphi(z)+\frac{1}{r}\langle y-z, z-x\rangle \geq 0, \forall y \in C\right\}
$$

for all $x \in H$. Assume that either (A1) or (A2) holds. Then the following results hold:

(i) $T_{r}^{(\Theta, \varphi)}(x) \neq \phi$ for each $x \in H$ and $T_{r}^{(\Theta, \varphi)}$ is single-valued;

(ii) $T_{r}^{(\Theta, \varphi)}$ is firmly nonexpansive, i.e., for any $x, y \in H$,

$$
\left\|T_{r}^{(\Theta, \varphi)} x-T_{r}^{(\Theta, \varphi)} y\right\|^{2} \leq\left\langle T_{r}^{(\Theta, \varphi)} x-T_{r}^{(\Theta, \varphi)} y, x-y\right\rangle
$$

(iii) $F\left(T_{r}^{(\Theta, \varphi)}\right)=\operatorname{MEP}(\Theta, \varphi)$;

(iv) $\operatorname{MEP}(\Theta, \varphi)$ is closed and convex.

Remark 2.1 If $\varphi=0$, then $T_{r}^{(\Theta, \varphi)}$ is rewritten as $T_{r}^{\Theta}$.

By a similar argument as that in the proof of Lemma 2.1 in [12], we have the following result.

Lemma 2.2 Let $C$ be a nonempty closed convex subset of $H$. Let $G_{1}, G_{2}: C \times C \rightarrow \mathbb{R}$ be two bifunctions satisfying conditions $(\mathrm{H} 1)-(\mathrm{H} 4)$ and let the mappings $B_{1}, B_{2}: C \rightarrow H$ be $\beta_{1}$-inverse strongly monotone and $\beta_{2}$-inverse strongly monotone, respectively. Then, for given $\bar{x}, \bar{y} \in C,(\bar{x}, \bar{y})$ is a solution of (1.4) if and only if $\bar{x}$ is a fixed point of the mapping $\Gamma: C \rightarrow C$ defined by

$$
\Gamma(x)=T_{\mu_{1}}^{G_{1}}\left[T_{\mu_{2}}^{G_{2}}\left(x-\mu_{2} B_{2} x\right)-\mu_{1} B_{1} T_{\mu_{2}}^{G_{2}}\left(x-\mu_{2} B_{2} x\right)\right], \quad \forall x \in C,
$$

where $\bar{y}=T_{\mu_{2}}^{G_{2}}\left(\bar{x}-\mu_{2} B_{2} \bar{x}\right)$.

The set of fixed points of the mapping $\Gamma$ is denoted by $\Omega$. 
Proposition 2.1 [3] Let $C, H, \Theta, \varphi$ and $T_{r}^{(\Theta, \varphi)}$ be as in Lemma 2.1. Then the following holds:

$$
\left\|T_{s}^{(\Theta, \varphi)} x-T_{t}^{(\Theta, \varphi)} x\right\|^{2} \leq \frac{s-t}{s}\left\langle T_{s}^{(\Theta, \varphi)} x-T_{t}^{(\Theta, \rho)} x, T_{s}^{(\Theta, \varphi)} x-x\right\rangle
$$

for all $s, t>0$ and $x \in H$.

Lemma 2.3 [21] Assume that $T$ is a nonexpansive self-mapping of a nonempty closed convex subset $C$ of $H$. If $T$ has a fixed point, then $I-T$ is demi-closed; that is, when $\left\{x_{n}\right\}$ is a sequence in $C$ converging weakly to some $x \in C$ and the sequence $\left\{(I-T) x_{n}\right\}$ converges strongly to some $y$, it follows that $(I-T) x=y$.

Lemma 2.4 [22] Assume that $\left\{a_{n}\right\}$ is a sequence of nonnegative real numbers such that

$$
a_{n+1} \leq\left(1-\gamma_{n}\right) a_{n}+\delta_{n}, \quad \forall n \geq 1
$$

where $\left\{\gamma_{n}\right\}$ is a sequence in $(0,1)$ and $\left\{\delta_{n}\right\}$ is a sequence such that

(i) $\sum_{n=1}^{\infty} \gamma_{n}=\infty$;

(ii) $\lim \sup _{n \rightarrow \infty} \frac{\delta_{n}}{\gamma_{n}} \leq 0$ or $\sum_{n=1}^{\infty}\left|\delta_{n}\right|<\infty$.

Then $\lim _{n \rightarrow \infty} a_{n}=0$.

Lemma 2.5 [17] Assume A is a strong positive linear bounded operator on a Hilbert space $H$ with a coefficient $\bar{\gamma}>0$ and $0<\rho \leq\|A\|^{-1}$. Then $\|I-\rho A\| \leq 1-\rho \bar{\gamma}$.

The following lemma is an immediate consequence of an inner product.

Lemma 2.6 In a real Hilbert space $H$, the following inequality holds:

$$
\|x+y\|^{2} \leq\|x\|^{2}+2\langle y, x+y\rangle
$$

for all $x, y \in H$.

\section{Main results}

Now we state and prove our main results.

Theorem 3.1 Let $C$ be a nonempty closed convex subset of a real Hilbert space $H$. Let $\Theta, G_{1}, G_{2}: C \times C \rightarrow \mathbb{R}$ be three bifunctions which satisfy assumptions (H1)-(H4) and $\varphi: C \rightarrow \mathbb{R}$ be a lower semicontinuous and convex function with assumption (A1) or (A2). Let the mappings $F, B_{1}, B_{2}: C \rightarrow H$ be $\zeta$-inverse strongly monotone, $\beta_{1}$-inverse strongly monotone and $\beta_{2}$-inverse strongly monotone, respectively. Let $T_{1}, T_{2}, \ldots, T_{N}$ be a finite family of nonexpansive mappings of $C$ into $H$ such that $\mathfrak{F}=\bigcap_{i=1}^{N} F\left(T_{i}\right) \cap$ GMEP $\cap \Omega \neq \phi$. Let $f$ be a contraction of $C$ into itself with a constant $\alpha(0<\alpha<1)$ and let $A$ be a strongly positive linear bounded operator with a coefficient $\bar{\gamma}>0$ such that $\|A\| \leq 1$. Assume that $0<\gamma<\frac{\bar{\gamma}}{\alpha}$. Let $x_{1} \in C$ and let $\left\{x_{n}\right\}$ be a sequence defined by

$$
\left\{\begin{array}{l}
z_{n}=T_{\delta_{n}}^{(\Theta, \varphi)}\left(x_{n}-\delta_{n} F x_{n}\right), \\
y_{n}=T_{\mu_{1}}^{G_{1}}\left[T_{\mu_{2}}^{G_{2}}\left(z_{n}-\mu_{2} B_{2} z_{n}\right)-\mu_{1} B_{1} T_{\mu_{2}}^{G_{2}}\left(z_{n}-\mu_{2} B_{2} z_{n}\right)\right] \\
x_{n+1}=\alpha_{n} \gamma f\left(W_{n} x_{n}\right)+\left(1-\alpha_{n} A\right) W_{n} y_{n}, \quad \forall n \geq 1
\end{array}\right.
$$


where $\alpha_{n} \in[0,1], \mu_{1} \in\left(0,2 \beta_{1}\right), \mu_{2} \in\left(0,2 \beta_{2}\right)$ and $\left\{\delta_{n}\right\} \subset[0,2 \zeta]$ satisfy the following conditions:

(i) $\lim _{n \rightarrow \infty} \alpha_{n}=0, \sum_{n=1}^{\infty} \alpha_{n}=0$ and $\sum_{n=1}^{\infty}\left|\alpha_{n+1}-\alpha_{n}\right|<\infty$;

(ii) $0<\liminf _{n \rightarrow \infty} \delta_{n} \leq \limsup \sup _{n \rightarrow \infty} \delta_{n}<2 \zeta$ and $\sum_{n=1}^{\infty}\left|\delta_{n+1}-\delta_{n}\right|<\infty$;

(iii) $\lim _{n \rightarrow \infty} \lambda_{n, i}=0$ and $\sum_{n=1}^{\infty}\left|\lambda_{n, i}-\lambda_{n-1, i}\right|<\infty$ for all $i=1,2, \ldots, N$.

Then $\left\{x_{n}\right\}$ converges strongly to $x^{*}=P_{\mathfrak{F}}(\gamma f+(I-A))\left(x^{*}\right)$, which solves the following variational inequality:

$$
\left\langle(A-\gamma f) x^{*}, x-x^{* \prime}\right| \geq 0, \quad \forall x \in \mathfrak{F},
$$

and $\left(x^{*}, y^{*}\right)$ is a solution of problem (1.4), where $y^{\prime \prime}=T_{\mu_{2}}^{G_{2}}\left(x^{*}-\mu_{2} B_{2} x^{*}\right)$.

Proof We divide the proof into several steps.

Step 1. $\left\{x_{n}\right\}$ is bounded.

Indeed, take $p \in \mathfrak{F}=\bigcap_{i=1}^{N} F\left(T_{i}\right) \cap \operatorname{GMEP} \cap \Omega \neq \phi$ arbitrarily. Since $p=T_{\delta_{n}}^{(\Theta, \varphi)}\left(p-\delta_{n} F p\right)$,

$F$ is $\zeta$-inverse strongly monotone and $0 \leq \delta_{n} \leq 2 \zeta$, we obtain that for any $n \geq 1$,

$$
\begin{aligned}
\left\|z_{n}-p\right\|^{2} & =\left\|T_{\delta_{n}}^{(\Theta, \varphi)}\left(x_{n}-\delta_{n} F x_{n}\right)-T_{\delta_{n}}^{(\Theta, \varphi)}\left(p-\delta_{n} F p\right)\right\|^{2} \\
& \leq\left\|\left(x_{n}-p\right)-\delta_{n}\left(F x_{n}-F p\right)\right\|^{2} \\
& =\left\|x_{n}-p\right\|^{2}-2 \delta_{n}\left\langle x_{n}-p, F x_{n}-F p\right\rangle+\delta_{n}^{2}\left\|F x_{n}-F p\right\|^{2} \\
& \leq\left\|x_{n}-p\right\|^{2}-2 \delta_{n} \zeta\left\|F x_{n}-F p\right\|^{2}+\delta_{n}^{2}\left\|F x_{n}-F p\right\|^{2} \\
& =\left\|x_{n}-p\right\|^{2}+\delta_{n}\left(\delta_{n}-2 \zeta\right)\left\|F x_{n}-F p\right\|^{2} \\
& \leq\left\|x_{n}-p\right\|^{2} .
\end{aligned}
$$

Putting $u_{n}=T_{\mu_{2}}^{G_{2}}\left(z_{n}-\mu_{2} B_{2} z_{n}\right)$ and $u=T_{\mu_{2}}^{G_{2}}\left(p-\mu_{2} B_{2} p\right)$, we have

$$
\begin{aligned}
\left\|u_{n}-u\right\|^{2} & =\left\|T_{\mu_{2}}^{G_{2}}\left(z_{n}-\mu_{2} B_{2} z_{n}\right)-T_{\mu_{2}}^{G_{2}}\left(p-\mu_{2} B_{2} p\right)\right\|^{2} \\
& \leq\left\|\left(z_{n}-p\right)-\mu_{2}\left(B_{2} z_{n}-B_{2} p\right)\right\|^{2} \\
& =\left\|z_{n}-p\right\|^{2}-2 \mu_{2}\left\langle z_{n}-p, B_{2} z_{n}-B_{2} p\right\rangle+\mu_{2}^{2}\left\|B_{2} z_{n}-B_{2} p\right\|^{2} \\
& \leq\left\|z_{n}-p\right\|^{2}-2 \mu_{2} \beta_{2}\left\|B_{2} z_{n}-B_{2} p\right\|^{2}+\mu_{2}^{2}\left\|B_{2} z_{n}-B_{2} p\right\|^{2} \\
& =\left\|z_{n}-p\right\|^{2}+\mu_{2}\left(\mu_{2}-2 \beta_{2}\right)\left\|B_{2} z_{n}-B_{2} p\right\|^{2} \\
& \leq\left\|z_{n}-p\right\|^{2} .
\end{aligned}
$$

And since $p=T_{\mu_{1}}^{G_{1}}\left(u-\mu_{1} B_{1} u\right)$, we know that for any $n \geq 1$,

$$
\begin{aligned}
\left\|y_{n}-p\right\|^{2} & =\left\|T_{\mu_{1}}^{G_{1}}\left(u_{n}-\mu_{1} B_{1} u_{n}\right)-T_{\mu_{1}}^{G_{1}}\left(u-\mu_{1} B_{1} u\right)\right\|^{2} \\
& \leq\left\|\left(u_{n}-u\right)-\mu_{1}\left(B_{1} u_{n}-B_{1} u\right)\right\|^{2} \\
& \leq\left\|u_{n}-u\right\|^{2}-2 \mu_{1}\left\langle u_{n}-u, B_{1} u_{n}-B_{1} u\right\rangle+\mu_{1}^{2}\left\|B_{1} u_{n}-B_{1} u\right\|^{2} \\
& \leq\left\|u_{n}-u\right\|^{2}-2 \mu_{1} \beta_{1}\left\|B_{1} u_{n}-B_{1} u\right\|+\mu_{1}\left\|B_{1} u_{n}-b_{1} u\right\|^{2} \\
& \leq\left\|u_{n}-u\right\|^{2}+\mu_{1}\left(\mu_{1}-2 \beta_{1}\right)\left\|B_{1} u_{n}-B_{1} u\right\|
\end{aligned}
$$




$$
\begin{aligned}
& \leq\left\|u_{n}-u\right\|^{2} \\
& \leq\left\|z_{n}-p\right\|^{2} .
\end{aligned}
$$

Furthermore, from (3.1), we have

$$
\begin{aligned}
\left\|x_{n+1}-p\right\| & =\left\|\alpha_{n} \gamma f\left(W_{n} x_{n}\right)+\left(1-\alpha_{n} A\right) W_{n} y_{n}-p\right\| \\
& =\left\|\alpha_{n}\left(\gamma f\left(W_{n} x_{n}\right)-A p\right)+\left(1-\alpha_{n} A\right)\left(W_{n} y_{n}-p\right)\right\| \\
& \leq \alpha_{n}\left\|\gamma f\left(W_{n} x_{n}\right)-A p\right\|+\left\|I-\alpha_{n} A\right\|\left\|W_{n} y_{n}-p\right\| \\
& \leq \alpha_{n}\left(\gamma\left\|f\left(W_{n} x_{n}\right)-f(p)\right\|+\|\gamma f(p)-A p\|\right)+\left(1-\alpha_{n} \bar{\gamma}\right)\left\|y_{n}-p\right\| \\
& \leq \alpha_{n}\left(\gamma \alpha\left\|x_{n}-p\right\|+\|\gamma f(p)-A p\|\right)+\left(1-\alpha_{n} \bar{\gamma}\right)\left\|x_{n}-p\right\| \\
& \leq\left[1-(\bar{\gamma}-\gamma \alpha) \alpha_{n}\right]\left\|x_{n}-p\right\|+\alpha_{n}\|\gamma f(p)-A p\| .
\end{aligned}
$$

By induction, we obtain that for all $n \geq 1$,

$$
\left\|x_{n}-p\right\| \leq \max \left\{\left\|x_{1}-p\right\|, \frac{1}{\bar{\gamma}-\gamma \alpha}\|\gamma f(p)-A p\|\right\} .
$$

Hence $\left\{x_{n}\right\}$ is bounded. Consequently, we deduce immediately that $\left\{z_{n}\right\},\left\{y_{n}\right\},\left\{f\left(W_{n} x_{n}\right)\right\}$ and $\left\{W_{n}\left(y_{n}\right)\right\}$ are bounded.

Step 2. $\lim _{n \rightarrow \infty}\left\|W_{n+1} y_{n}-W_{n} y_{n}\right\|=0$.

It follows from the definition of $W_{n}$ that

$$
\begin{aligned}
\left\|W_{n+1} y_{n}-W_{n} y_{n}\right\| \\
=\left\|\lambda_{n+1, N} T_{N} U_{n+1, N-1} y_{n}-\left(1-\lambda_{n+1, N}\right) y_{n}-\lambda_{n, N} T_{N} U_{n, N-1} y_{n}-\left(1-\lambda_{n, N}\right) y_{n}\right\| \\
\leq\left|\lambda_{n+1, N}-\lambda_{n, N}\right|\left\|y_{n}\right\|+\left\|\lambda_{n+1, N} T_{N} U_{n+1, N-1} y_{n}-\lambda_{n, N} T_{N} U_{n, N-1} y_{n}\right\| \\
\leq\left|\lambda_{n+1, N}-\lambda_{n, N}\right|\left\|y_{n}\right\|+\left\|\lambda_{n+1, N}\left(T_{N} U_{n+1, N-1} y_{n}-T_{N} U_{n, N-1} y_{n}\right)\right\| \\
\quad+\left|\lambda_{n+1, N}-\lambda_{n, N}\right|\left\|T_{N} U_{n, N-1} y_{n}\right\| \\
\leq\left|\lambda_{n+1, N}-\lambda_{n, N}\right|\left\|y_{n}\right\|+\lambda_{n+1, N}\left\|U_{n+1, N-1} y_{n}-U_{n, N-1} y_{n}\right\| \\
\quad+\left|\lambda_{n+1, N}-\lambda_{n, N}\right|\left\|T_{N} U_{n, N-1} y_{n}\right\| .
\end{aligned}
$$

Since $\left\{y_{n}\right\}$ is bounded and $T_{k}, U_{n, k}$ are nonexpansive, $\lim _{n \rightarrow \infty}\left\|W_{n+1} y_{n}-W_{n} y_{n}\right\|=0$.

Step 3. $\lim _{n \rightarrow \infty}\left\|x_{n+1}-x_{n}\right\|=0$.

We estimate $\left\|y_{n+1}-y_{n}\right\|,\left\|W_{n+1} x_{n+1}-W_{n} x_{n}\right\|$ and $\left\|W_{n+1} y_{n+1}-W_{n} y_{n}\right\|$. From (3.1) we have

$$
\begin{aligned}
\left\|y_{n+1}-y_{n}\right\|^{2}= & \left\|T_{\mu_{1}}^{G_{1}}\left(u_{n+1}-\mu_{1} B_{1} u_{n+1}\right)-T_{\mu_{1}}^{G_{1}}\left(u_{n}-\mu_{1} B_{1} u_{n}\right)\right\|^{2} \\
& \leq\left\|\left(u_{n+1}-u_{n}\right)-\mu_{1}\left(B_{1} u_{n+1}-B_{1} u_{n}\right)\right\|^{2} \\
& \leq\left\|u_{n+1}-u_{n}\right\|^{2}+\mu_{1}\left(\mu_{1}-2 \beta_{1}\right)\left\|B_{1} u_{n+1}-B_{1} u_{n}\right\|^{2} \\
& \leq\left\|u_{n+1}-u_{n}\right\|^{2} \\
& =\left\|T_{\mu_{2}}^{G_{2}}\left(z_{n+1}-\mu_{2} B_{2} z_{n+1}\right)-T_{\mu_{2}}^{G_{2}}\left(z_{n}-\mu_{2} B_{2} z_{n}\right)\right\|^{2} \\
& \leq\left\|\left(z_{n+1}-z_{n}\right)-\mu_{2}\left(B_{2} z_{n+1}-B_{2} z_{n}\right)\right\|^{2}
\end{aligned}
$$




$$
\begin{aligned}
& \leq\left\|z_{n+1}-z_{n}\right\|^{2}+\mu_{2}\left(\mu_{2}-2 \beta_{2}\right)\left\|B_{2} z_{n+1}-B_{2} z_{n}\right\|^{2} \\
& \leq\left\|z_{n+1}-z_{n}\right\|^{2}, \\
& \left\|\left(x_{n+1}-\delta_{n+1} F x_{n+1}\right)-\left(x_{n}-\delta_{n} F x_{n}\right)\right\| \\
& \leq\left\|x_{n+1}-x_{n}-\delta_{n+1}\left(F x_{n+1}-F x_{n}\right)\right\|+\left|\delta_{n}-\delta_{n+1}\right|\left\|F x_{n}\right\| \\
& \leq\left\|x_{n+1}-x_{n}\right\|+\left|\delta_{n}-\delta_{n+1}\right|\left\|F x_{n}\right\|
\end{aligned}
$$

and

$$
\begin{aligned}
& \left\|z_{n+1}-z_{n}\right\| \\
& =\left\|T_{\delta_{n+1}}^{(\Theta, \varphi)}\left(x_{n+1}-\delta_{n+1} F x_{n+1}\right)-T_{\delta_{n}}^{(\Theta, \varphi)}\left(x_{n}-\delta_{n} F x_{n}\right)\right\| \\
& \leq\left\|T_{\delta_{n+1}}^{(\Theta, \varphi)}\left(x_{n+1}-\delta_{n+1} F x_{n+1}\right)-T_{\delta_{n+1}}^{(\Theta, \varphi)}\left(x_{n}-\delta_{n} F x_{n}\right)\right\| \\
& +\left\|T_{\delta_{n+1}}^{(\Theta, \varphi)}\left(x_{n}-\delta_{n} F x_{n}\right)-T_{\delta_{n}}^{(\Theta, \varphi)}\left(x_{n}-\delta_{n} F x_{n}\right)\right\| \\
& \leq\left\|\left(x_{n+1}-\delta_{n+1} F x_{n+1}\right)-\left(x_{n}-\delta_{n} F x_{n}\right)\right\| \\
& +\left\|T_{\delta_{n+1}}^{(\Theta, \varphi)}\left(x_{n}-\delta_{n} F x_{n}\right)-T_{\delta_{n}}^{(\Theta, \varphi)}\left(x_{n}-\delta_{n} F x_{n}\right)\right\| \\
& \leq\left\|x_{n+1}-x_{n}\right\|+\left|\delta_{n+1}-\delta_{n}\right||| F x_{n} \| \\
& +\left\|T_{\delta_{n+1}}^{(\Theta, \varphi)}\left(x_{n}-\delta_{n} F x_{n}\right)-T_{\delta_{n}}^{(\Theta, \varphi)}\left(x_{n}-\delta_{n} F x_{n}\right)\right\| .
\end{aligned}
$$

It follows from (3.5) and (3.6) that

$$
\begin{aligned}
\left\|y_{n+1}-y_{n}\right\| \leq & \left\|z_{n+1}-z_{n}\right\| \\
\leq & \left\|x_{n+1}-x_{n}\right\|+\left|\delta_{n+1}-\delta_{n}\right|\left\|F x_{n}\right\| \\
& \quad\left\|T_{\delta_{n+1}}^{(\Theta, \varphi)}\left(x_{n}-\delta_{n} F x_{n}\right)-T_{\delta_{n}}^{(\Theta, \varphi)}\left(x_{n}-\delta_{n} F x_{n}\right)\right\| .
\end{aligned}
$$

Without loss of generality, let us assume that there exists a real number $a$ such that $\delta_{n}>$ $a>0$ for all $n$. Utilizing Proposition 2.1, we have

$$
\begin{aligned}
& \left\|T_{\delta_{n+1}}^{(\Theta, \varphi)}\left(x_{n}-\delta_{n} F x_{n}\right)-T_{\delta_{n}}^{(\Theta, \varphi)}\left(x_{n}-\delta_{n} F x_{n}\right)\right\| \\
& \quad \leq \frac{\left|\delta_{n+1}-\delta_{n}\right|}{\delta_{n+1}}\left\|T_{\delta_{n+1}}^{(\Theta, \varphi)}\left(I-\delta_{n} F\right) x_{n}\right\| \\
& \leq \frac{\left|\delta_{n+1}-\delta_{n}\right|}{a}\left\|T_{\delta_{n+1}}^{(\Theta, \varphi)}\left(I-\delta_{n} F\right) x_{n}\right\| .
\end{aligned}
$$

It follows from the definition of $W_{n}$ that

$$
\begin{aligned}
\| & W_{n+1} y_{n}-W_{n} y_{n} \| \\
= & \left\|\lambda_{n+1, N} T_{N} U_{n+1, N-1} y_{n}+\left(1-\lambda_{n+1, N}\right) y_{n}-\lambda_{n, N} T_{N} U_{n, N-1} y_{n}-\left(1-\lambda_{n, N}\right) y_{n}\right\| \\
\leq & \left|\lambda_{n+1, N}-\lambda_{n, N}\right|\left\|y_{n}\right\|+\left\|\lambda_{n+1, N} T_{N} U_{n+1, N-1} y_{n}-\lambda_{n, N} T_{N} U_{n, N-1} y_{n}\right\| \\
\leq & \left|\lambda_{n+1, N}-\lambda_{n, N}\right|\left\|y_{n}\right\|+\left\|\lambda_{n+1, N}\left(T_{N} U_{n+1, N-1} y_{n}-T_{N} U_{n, N-1} y_{n}\right)\right\| \\
& \quad+\left|\lambda_{n+1, N}-\lambda_{n, N}\right|\left\|T_{N} U_{n, N-1} y_{n}\right\|
\end{aligned}
$$




$$
\begin{aligned}
\leq & \left|\lambda_{n+1, N}-\lambda_{n, N}\right|\left\|y_{n}\right\|+\lambda_{n+1, N}\left\|U_{n+1, N-1} y_{n}-U_{n, N-1} y_{n}\right\| \\
& +\left|\lambda_{n+1, N}-\lambda_{n, N}\right|\left\|T_{N} U_{n, N-1} y_{n}\right\| \\
\leq & M_{1}\left|\lambda_{n+1, N}-\lambda_{n, N}\right|+\lambda_{n+1, N}\left\|U_{n+1, N-1} y_{n}-U_{n, N-1} y_{n}\right\|
\end{aligned}
$$

where $M_{1}$ is a constant such that $M_{1} \geq 2 \max \left\{\sup _{n \geq 1}\left\|y_{n}\right\|, \sup _{n \geq 1}\left\|T_{N} U_{n, N-1} y_{n}\right\|\right\}$. Next, we consider

$$
\begin{aligned}
\left\|U_{n+1, N-1} y_{n}-U_{n, N-1} y_{n}\right\| \\
=\| \lambda_{n+1, N-1} T_{N-1} U_{n+1, N-2} y_{n}+\left(1-\lambda_{n+1, N-1}\right) y_{n} \\
\quad-\lambda_{n, N-1} T_{N-1} U_{n, N-2} y_{n}-\left(1-\lambda_{n, N-1}\right) y_{n} \| \\
\leq\left|\lambda_{n+1, N-1}-\lambda_{n, N-1}\right|\left\|y_{n}\right\|+\left\|\lambda_{n+1, N-1} T_{N-1} U_{n+1, N-2} y_{n}-\lambda_{n, N-1} T_{N-1} U_{n, N-2} y_{n}\right\| \\
\leq\left|\lambda_{n+1, N-1}-\lambda_{n, N-1}\right|\left\|y_{n}\right\|+\lambda_{n+1, N-1}\left\|T_{N-1} U_{n+1, N-2} y_{n}-T_{N-1} U_{n, N-2} y_{n}\right\| \\
\quad+\left|\lambda_{n+1, N-1}-\lambda_{n, N-1}\right|\left\|T_{N-1} U_{n, N-2} y_{n}\right\| \\
\leq M_{2}\left|\lambda_{n+1, N-1}-\lambda_{n, N-1}\right|+\left\|U_{n+1, N-2} y_{n}-U_{n, N-2} y_{n}\right\|
\end{aligned}
$$

where $M_{2}$ is a constant that $M_{2} \geq 2 \max \left\{\sup _{n \geq 1}\left\|y_{n}\right\|, \sup _{n \geq 1}\left\|T_{N-1} U_{n, N-2} y_{n}\right\|\right\}$. In a similar way, we obtain

$$
\left\|U_{n+1, N-1} y_{n}-U_{n, N-1} y_{n}\right\| \leq M_{3} \sum_{i=1}^{N-1}\left|\lambda_{n+1, i}-\lambda_{n, i}\right|
$$

where $M_{3}$ is an appropriate constant. Substituting (3.10) into (3.9), we have that

$$
\begin{aligned}
\left\|W_{n+1} y_{n}-W_{n} y_{n}\right\| & \leq M_{1}\left|\lambda_{n+1, N}-\lambda_{n, N}\right|+\lambda_{n+1, N} M_{3} \sum_{i=1}^{N-1}\left|\lambda_{n+1, i}-\lambda_{n, i}\right| \\
& \leq M_{4} \sum_{i=1}^{N}\left|\lambda_{n+1, i}-\lambda_{n, i}\right|
\end{aligned}
$$

where $M_{4}$ is a constant such that $M_{4} \geq \max \left\{M_{1}, M_{3}\right\}$. Similarly, we have

$$
\left\|W_{n+1} x_{n}-W_{n} x_{n}\right\| \leq M_{5} \sum_{i=1}^{N}\left|\lambda_{n+1, i}-\lambda_{n, i}\right|
$$

where $M_{5}$ is an appropriate constant. Hence it follows from (3.1), (3.7), (3.8), (3.11) and (3.12) that

$$
\begin{aligned}
& \left\|x_{n+2}-x_{n+1}\right\| \\
& =\|\left(I-\alpha_{n+1} A\right)\left(W_{n+1} y_{n+1}-W_{n} y_{n}\right)-\left(\alpha_{n+1}-\alpha_{n}\right) A W_{n} y_{n} \\
& \quad+\gamma\left[\alpha_{n+1}\left(f\left(W_{n+1} x_{n+1}\right)-f\left(W_{n} x_{n}\right)\right)+f\left(W_{n} x_{n}\right)\left(\alpha_{n+1}-\alpha_{n}\right)\right] \| \\
& \leq\left(1-\alpha_{n+1} \bar{\gamma}\right)\left(\left\|W_{n+1} y_{n+1}-W_{n+1} y_{n}\right\|+\left\|W_{n+1} y_{n}-W_{n} y_{n}\right\|\right) \\
& \quad+\left|\alpha_{n+1}-\alpha_{n}\right|\left\|A W_{n} y_{n}\right\|
\end{aligned}
$$




$$
\begin{aligned}
& +\gamma\left[\alpha_{n+1}\left\|f\left(W_{n+1} x_{n+1}\right)-f\left(W_{n} x_{n}\right)\right\|+f\left(W_{n} x_{n}\right)\left(\alpha_{n+1}-\alpha_{n}\right)\right] \\
& \leq\left(1-\alpha_{n+1} \bar{\gamma}\right)\left(\left\|y_{n+1}-y_{n}\right\|+\left\|W_{n+1} y_{n}-W_{n} y_{n}\right\|\right) \\
& +\left|\alpha_{n+1}-\alpha_{n}\right|\left\|A W_{n} y_{n}\right\| \\
& +\gamma\left[\alpha_{n+1} \alpha\left(\left\|x_{n+1}-x_{n}\right\|+\left\|W_{n+1} x_{n}-W_{n} x_{n}\right\|\right)+\left|\alpha_{n+1}-\alpha_{n}\right|\left\|f\left(W_{n} x_{n}\right)\right\|\right] \\
& \leq\left(1-\alpha_{n+1} \bar{\gamma}\right)\left(\left\|x_{n+1}-x_{n}\right\|+\left|\delta_{n+1}-\delta_{n}\right|\left\|F x_{n}\right\|\right. \\
& \left.+\left\|T_{\delta_{n+1}}^{(\Theta, \varphi)}\left(x_{n}-\delta_{n} F x_{n}\right)-T_{\delta_{n}}^{(\Theta, \varphi)}\left(x_{n}-\delta_{n} F x_{n}\right)\right\|+M_{4} \sum_{i=1}^{N}\left|\lambda_{n+1, i}-\lambda_{n, i}\right|\right) \\
& +\left|\alpha_{n+1}-\alpha_{n}\right|\left\|A W_{n} y_{n}\right\| \\
& +\gamma \alpha \alpha_{n+1}\left\|x_{n+1}-x_{n}\right\|+\gamma \alpha \alpha_{n+1}\left\|W_{n+1} x_{n}-W_{n} x_{n}\right\| \\
& +\gamma\left|\alpha_{n+1}-\alpha_{n}\right|\left\|f\left(W_{n} x_{n}\right)\right\| \\
& \leq\left[1-\alpha_{n+1}(\bar{\gamma}-\gamma \alpha)\right]\left\|x_{n+1}-x_{n}\right\|+\left|\delta_{n+1}-\delta_{n}\right|\left\|F x_{n}\right\| \\
& +\frac{\left|\delta_{n+1}-\delta_{n}\right|}{a}\left\|T_{\delta_{n+1}}^{(\Theta, \varphi)}\left(I-\delta_{n} F\right) x_{n}\right\|+M_{4} \sum_{i=1}^{N}\left|\lambda_{n+1, i}-\lambda_{n, i}\right| \\
& +\left|\alpha_{n+1}-\alpha_{n}\right|\left\|A W_{n} y_{n}\right\|+\gamma \alpha \alpha_{n+1} M_{5} \sum_{i=1}^{N}\left|\lambda_{n+1, i}-\lambda_{n, i}\right| \\
& +\gamma\left|\alpha_{n+1}-\alpha_{n}\right|\left\|f\left(W_{n} x_{n}\right)\right\| \\
& \leq\left[1-\alpha_{n+1}(\bar{\gamma}-\gamma \alpha)\right]\left\|x_{n+1}-x_{n}\right\| \\
& +M_{6}\left[2\left|\delta_{n+1}-\delta_{n}\right|+\sum_{i=1}^{N}\left|\lambda_{n+1, i}-\lambda_{n, i}\right|+(1+\gamma)\left|\alpha_{n+1}-\alpha_{n}\right|\right],
\end{aligned}
$$

where $M_{6}$ is a constant such that $M_{6} \geq \max \left\{\sup _{n \geq 1}\left\|F x_{n}\right\|, \frac{1}{a} \sup _{n \geq 1}\left\|T_{\delta_{n+1}}^{(\Theta, \varphi)}\left(I-\delta_{n} F\right) x_{n}\right\|\right.$, $\left.M_{4}+\gamma M_{5}, \sup _{n \geq 1}\left\|A W_{n} y_{n}\right\|, \sup _{n \geq 1}\left\|f\left(W_{n} x_{n}\right)\right\|\right\}$. By Lemma 2.4 , we get $\lim _{n \rightarrow \infty} \| x_{n+1}-$ $x_{n} \|=0$.

Step 4. $\lim _{n \rightarrow \infty}\left\|F x_{n}-F p\right\|=0, \lim _{n \rightarrow \infty}\left\|B_{1} u_{n}-B_{1} u\right\|=0$ and $\lim _{n \rightarrow \infty}\left\|B_{2} z_{n}-B_{2} p\right\|=0$. Indeed, from (3.1)-(3.4) we get

$$
\begin{aligned}
&\left\|x_{n+1}-p\right\|^{2} \\
&=\left\|\alpha_{n}\left(\gamma f\left(W_{n} x_{n}\right)-A p\right)+\left(I-\alpha_{n} A\right)\left(W_{n} y_{n}-p\right)\right\|^{2} \\
& \leq\left(\alpha_{n}\left\|\gamma f\left(W_{n} x_{n}\right)-A p\right\|+\left(1-\alpha_{n} \bar{\gamma}\right)\left\|y_{n}-p\right\|\right)^{2} \\
& \leq \alpha_{n}\left\|\gamma f\left(W_{n} x_{n}\right)-A p\right\|^{2}+\left(1-\alpha_{n} \bar{\gamma}\right)\left\|y_{n}-p\right\|^{2} \\
&+2 \alpha_{n}\left\|\gamma f\left(W_{n} x_{n}\right)-A p\right\|\left\|y_{n}-p\right\| \\
& \leq \alpha_{n}\left\|\gamma f\left(W_{n} x_{n}\right)-A p\right\|^{2}+\left(1-\alpha_{n} \bar{\gamma}\right)\left[\left\|u_{n}-u\right\|^{2}+\mu_{1}\left(\mu_{1}-2 \beta_{1}\right)\left\|B_{1} u_{n}-B_{1} u\right\|^{2}\right] \\
&+2 \alpha_{n}\left\|\gamma f\left(W_{n} x_{n}\right)-A p\right\|\left\|y_{n}-p\right\| \\
& \leq \alpha_{n}\left\|\gamma f\left(W_{n} x_{n}\right)-A p\right\|^{2}+\left(1-\alpha_{n} \bar{\gamma}\right)\left[\left\|z_{n}-p\right\|^{2}+\mu_{2}\left(\mu_{2}-2 \beta_{2}\right)\left\|B_{2} z_{n}-B_{2} p\right\|^{2}\right.
\end{aligned}
$$




$$
\begin{aligned}
& \left.+\mu_{1}\left(\mu_{1}-2 \beta_{1}\right)\left\|B_{1} u_{n}-B_{1} u\right\|^{2}\right]+2 \alpha_{n}\left\|\gamma f\left(W_{n} x_{n}\right)-A p\right\|\left\|y_{n}-p\right\| \\
\leq & \alpha_{n}\left\|\gamma f\left(W_{n} x_{n}\right)-A p\right\|^{2}+\left\|x_{n}-p\right\|^{2}+\delta_{n}\left(\delta_{n}-2 \zeta\right)\left\|F_{n}-F p\right\|^{2} \\
& +\mu_{2}\left(\mu_{2}-2 \beta_{2}\right)\left\|B_{2} z_{n}-B_{2} p\right\|^{2}+\mu_{1}\left(\mu_{1}-2 \beta_{1}\right)\left\|B_{1} u_{n}-B_{1} u\right\|^{2} \\
& +2 \alpha_{n}\left\|\gamma f\left(W_{n} x_{n}\right)-A p\right\|\left\|y_{n}-p\right\| .
\end{aligned}
$$

Therefore

$$
\begin{aligned}
& \delta_{n}\left(2 \zeta-\delta_{n}\right)\left\|F x_{n}-F p\right\|^{2}+\mu_{2}\left(2 \beta_{2}-\mu_{2}\right)\left\|B_{2} z_{n}-B_{2} p\right\|^{2}+\mu_{1}\left(2 \beta_{1}-\mu_{1}\right)\left\|B_{1} u_{n}-B_{1} u\right\|^{2} \\
& \leq \alpha_{n}\left\|\gamma f\left(W_{n} x_{n}\right)-A p\right\|^{2}+\left\|x_{n}-p\right\|-\left\|x_{n+1}-p\right\|^{2}+2 \alpha_{n}\left\|\gamma f\left(W_{n} x_{n}\right)-A p\right\|\left\|y_{n}-p\right\| \\
& \leq \alpha_{n}\left\|\gamma f\left(W_{n} x_{n}\right)-A p\right\|^{2}+\left(\left\|x_{n}-p\right\|+\left\|x_{n+1}-p\right\|\right)\left\|x_{n}-x_{n+1}\right\| \\
& \quad+2 \alpha_{n}\left\|\gamma f\left(W_{n} x_{n}\right)-A p\right\|\left\|y_{n}-p\right\| .
\end{aligned}
$$

Since $\alpha_{n} \rightarrow 0$ and $\left\|x_{n}-x_{n+1}\right\| \rightarrow 0$ as $n \rightarrow \infty$, we have $\lim _{n \rightarrow \infty}\left\|F x_{n}-F p\right\|=0$, $\lim _{n \rightarrow \infty}\left\|B_{1} u_{n}-B_{1} u\right\|=0$ and $\lim _{n \rightarrow \infty}\left\|B_{2} z_{n}-B_{2} p\right\|=0$.

Step 5. $\lim _{n \rightarrow \infty}\left\|x_{n}-z_{n}\right\|=0, \lim _{n \rightarrow \infty}\left\|z_{n}-y_{n}\right\|=0$ and $\lim _{n \rightarrow \infty}\left\|x_{n}-y_{n}\right\|=0$.

Indeed, from (3.2), (3.3) and Lemma 2.1, we have

$$
\begin{aligned}
\left\|u_{n}-u\right\|^{2} & \left\|T_{\mu_{2}}^{G_{2}}\left(z_{n}-\mu_{2} B_{2} z_{n}\right)-T_{\mu_{2}}^{G_{2}}\left(p-\mu_{2} B_{2} p\right)\right\|^{2} \\
\leq & \left\langle\left(z_{n}-\mu_{2} B_{2} z_{n}\right)-\left(p-\mu_{2} B_{2} p\right), u_{n}-u\right\rangle \\
= & \frac{1}{2}\left[\left\|\left(z_{n}-\mu_{2} B_{2} z_{n}\right)-\left(p-\mu_{2} B_{2} p\right)\right\|^{2}+\left\|u_{n}-u\right\|^{2}\right. \\
& \left.\quad-\left\|\left(z_{n}-\mu_{2} B_{2} z_{n}\right)-\left(p-\mu_{2} B_{2} p\right)-\left(u_{n}-u\right)\right\|^{2}\right] \\
\leq & \frac{1}{2}\left[\left\|z_{n}-p\right\|^{2}+\left\|u_{n}-u\right\|^{2}-\left\|\left(z_{n}-u_{n}\right)-\mu_{2}\left(B_{2} z_{n}-B_{2} p\right)-(p-u)\right\|^{2}\right] \\
\leq & \frac{1}{2}\left[\left\|x_{n}-p\right\|^{2}+\left\|u_{n}-u\right\|^{2}-\left\|\left(z_{n}-u_{n}\right)-(p-u)\right\|^{2}\right. \\
& \left.+2 \mu_{2}\left\langle\left(z_{n}-u_{n}\right)-(p-u), B_{2} z_{n}-B_{2} p\right\rangle-\mu_{2}^{2}\left\|B_{2} z_{n}-B_{2} p\right\|^{2}\right]
\end{aligned}
$$

and

$$
\begin{aligned}
\left\|y_{n}-p\right\|^{2} & \\
= & \left\|T_{\mu_{1}}^{G_{1}}\left(u_{n}-\mu_{1} B_{1} u_{n}\right)-T_{\mu_{1}}^{G_{1}}\left(u-\mu_{1} B_{1} u\right)\right\|^{2} \\
\leq & \left\langle\left(u_{n}-\mu_{1} B_{1} u_{n}\right)-\left(u-\mu_{1} B_{1} u\right), y_{n}-p\right\rangle \\
= & \frac{1}{2}\left[\left\|\left(u_{n}-\mu_{1} B_{1} u_{n}\right)-\left(u-\mu_{1} B_{1} u\right)\right\|^{2}+\left\|y_{n}-p\right\|^{2}\right. \\
& \left.\quad-\left\|\left(u_{n}-\mu_{1} B_{1} u_{n}\right)-\left(u-\mu_{1} B_{1} u\right)-\left(y_{n}-p\right)\right\|^{2}\right] \\
\leq & \frac{1}{2}\left[\left\|u_{n}-u\right\|^{2}+\left\|y_{n}-p\right\|^{2}-\left\|\left(u_{n}-y_{n}\right)+(p-u)\right\|^{2}\right. \\
& \left.\quad+2 \mu_{1}\left\langle B_{1} u_{n}-B_{1} u,\left(u_{n}-y_{n}\right)+(p-u)\right\rangle-\mu_{1}^{2}\left\|B_{1} u_{n}-B_{1} u\right\|^{2}\right]
\end{aligned}
$$




$$
\begin{aligned}
\leq & \frac{1}{2}\left[\left\|x_{n}-p\right\|^{2}+\left\|y_{n}-p\right\|^{2}-\left\|\left(u_{n}-y_{n}\right)+(p-u)\right\|^{2}\right. \\
& \left.+2 \mu_{1}\left(B_{1} u_{n}-B_{1} u,\left(u_{n}-y_{n}\right)+(p-u)\right\rangle\right],
\end{aligned}
$$

which imply that

$$
\begin{aligned}
\left\|u_{n}-u\right\|^{2} \leq & \left\|x_{n}-p\right\|^{2}-\left\|\left(z_{n}-u_{n}\right)-(p-u)\right\|^{2} \\
& +2 \mu_{2}\left\langle\left(z_{n}-u_{n}\right)-(p-u), B_{2} z_{n}-B_{2} p\right\rangle-\mu_{2}^{2}\left\|B_{2} z_{n}-B_{2} p\right\|^{2}
\end{aligned}
$$

and

$$
\begin{aligned}
\left\|y_{n}-p\right\|^{2} \leq & \left\|x_{n}-p\right\|^{2}-\left\|\left(u_{n}-y_{n}\right)+(p-u)\right\|^{2} \\
& +2 \mu_{1}\left\|B_{1} u_{n}-B_{1} u\right\|\left\|\left(u_{n}-y_{n}\right)+(p-u)\right\| .
\end{aligned}
$$

It follows from (3.14) that

$$
\begin{aligned}
\left\|x_{n+1}-p\right\|^{2} \\
=\left\|\alpha_{n}\left(\gamma f\left(W_{n} x_{n}\right)-A p\right)+\left(I-\alpha_{n} A\right)\left(W_{n} y_{n}-p\right)\right\|^{2} \\
\leq \alpha_{n}\left\|\gamma f\left(W_{n} x_{n}\right)-A p\right\|^{2}+\left(1-\alpha_{n} \bar{\gamma}\right)\left\|y_{n}-p\right\|^{2} \\
\quad+2 \alpha_{n}\left\|\gamma f\left(W_{n} x_{n}\right)-A p\right\|\left\|y_{n}-p\right\| \\
\leq \quad \alpha_{n}\left\|\gamma f\left(W_{n} x_{n}\right)-A p\right\|^{2}+\left(1-\alpha_{n} \bar{\gamma}\right)\left[\left\|x_{n}-p\right\|^{2}-\left\|\left(u_{n}-y_{n}\right)+(p-u)\right\|^{2}\right. \\
\left.\quad+2 \mu_{1}\left\|B_{1} u_{n}-B_{1} u\right\|\left\|\left(u_{n}-y_{n}\right)+(p-u)\right\|\right]+2 \alpha_{n}\left\|\gamma f\left(W_{n} x_{n}\right)-A p\right\|\left\|y_{n}-p\right\|,
\end{aligned}
$$

which gives that

$$
\begin{aligned}
&\left(1-\alpha_{n} \bar{\gamma}\right)\left\|\left(u_{n}-y_{n}\right)+(p-u)\right\|^{2} \\
& \leq \alpha_{n}\left\|\gamma f\left(W_{n} x_{n}\right)-A p\right\|^{2}+\left\|x_{n}-p\right\|^{2}-\left\|x_{n+1}-p\right\|^{2} \\
&+2 \mu_{1}\left(1-\alpha_{n} \bar{\gamma}\right)\left\|B_{1} u_{n}-B_{1} u\right\|\left\|\left(u_{n}-y_{n}\right)+(p-u)\right\| \\
&+2 \alpha_{n}\left\|\gamma f\left(W_{n} x_{n}\right)-A p\right\|\left\|y_{n}-p\right\| \\
& \leq \alpha_{n}\left\|\gamma f\left(W_{n} x_{n}\right)-A p\right\|^{2}+\left(\left\|x_{n}-p\right\|+\left\|x_{n+1}-p\right\|\right)\left\|x_{n}-x_{n+1}\right\| \\
&+2 \mu_{1}\left(1-\alpha_{n} \bar{\gamma}\right)\left\|B_{1} u_{n}-B_{1} u\right\|\left\|\left(u_{n}-y_{n}\right)+(p-u)\right\| \\
&+2 \alpha_{n}\left\|\gamma f\left(W_{n} x_{n}\right)-A p\right\|\left\|y_{n}-p\right\| .
\end{aligned}
$$

Since $\alpha_{n} \rightarrow 0,\left\|x_{n+1}-x_{n}\right\| \rightarrow 0$ and $\left\|B_{1} u_{n}-B_{1} u\right\| \rightarrow 0$ as $n \rightarrow \infty$, we have

$$
\lim _{n \rightarrow \infty}\left\|\left(u_{n}-y_{n}\right)+(p-u)\right\|^{2}=0 .
$$

Also, from (3.4) and (3.13), we have

$$
\begin{aligned}
& \left\|x_{n+1}-p\right\|^{2} \\
& \quad=\left\|\alpha_{n}\left(\gamma f\left(W_{n} x_{n}\right)-A p\right)+\left(I-\alpha_{n} A\right)\left(W_{n} y_{n}-p\right)\right\|^{2}
\end{aligned}
$$




$$
\begin{aligned}
\leq & \alpha_{n}\left\|\gamma f\left(W_{n} x_{n}\right)-A p\right\|^{2}+\left(1-\alpha_{n} \bar{\gamma}\right)\left\|y_{n}-p\right\|^{2} \\
& +2 \alpha_{n}\left\|\gamma f\left(W_{n} x_{n}\right)-A p\right\|\left\|y_{n}-p\right\| \\
\leq & \alpha_{n}\left\|\gamma f\left(W_{n} x_{n}\right)-A p\right\|^{2}+\left(1-\alpha_{n} \bar{\gamma}\right)\left\|u_{n}-u\right\|^{2} \\
& +2 \alpha_{n}\left\|\gamma f\left(W_{n} x_{n}\right)-A p\right\|\left\|y_{n}-p\right\| \\
\leq & \alpha_{n}\left\|\gamma f\left(W_{n} x_{n}\right)-A p\right\|^{2}+\left(1-\alpha_{n} \bar{\gamma}\right)\left[\left\|x_{n}-p\right\|^{2}-\left\|\left(z_{n}-u_{n}\right)-(p-u)\right\|^{2}\right. \\
& \left.+2 \mu_{2}\left(\left(z_{n}-u_{n}\right)-(p-u), B_{2} z_{n}-B_{2} p\right\rangle\right] \\
& +2 \alpha_{n}\left\|\gamma f\left(W_{n} x_{n}\right)-A p\right\|\left\|y_{n}-p\right\| .
\end{aligned}
$$

So, we have

$$
\begin{aligned}
\left(1-\alpha_{n} \bar{\gamma}\right)\left\|\left(z_{n}-u_{n}\right)-(p-u)\right\|^{2} \\
\leq \alpha_{n}\left\|\gamma f\left(W_{n} x_{n}\right)-A p\right\|^{2}+\left\|x_{n}-p\right\|^{2}-\left\|x_{n+1}-p\right\|^{2} \\
\quad+2 \mu_{2}\left\|\left(z_{n}-u_{n}\right)-(p-u)\right\|\left\|B_{2} z_{n}-B_{2} p\right\| \\
\left.\quad+2 \alpha_{n} \| \gamma f\left(W_{n} x_{n}\right)-A p\right)\|\| y_{n}-p \| \\
\leq \alpha_{n}\left\|\gamma f\left(W_{n} x_{n}\right)-A p\right\|^{2}+\left(\left\|x_{n}-p\right\|+\left\|x_{n+1}-p\right\|\right)\left\|x_{n}-x_{n+1}\right\| \\
\quad+2 \mu_{2}\left\|\left(z_{n}-u_{n}\right)-(p-u)\right\|\left\|B_{2} z_{n}-B_{2} p\right\| \\
\quad+2 \alpha_{n}\left\|\gamma f\left(W_{n} x_{n}\right)-A p\right\|\left\|y_{n}-p\right\| .
\end{aligned}
$$

Note that $\left\|B_{2} z_{n}-B_{2} p\right\| \rightarrow 0$ as $n \rightarrow \infty$. Then we have

$$
\lim _{n \rightarrow \infty}\left\|\left(z_{n}-u_{n}\right)-(p-u)\right\|=0 .
$$

In addition, from the firm nonexpansivity of $T_{\delta_{n}}^{(\Theta, \varphi)}$, we have

$$
\begin{aligned}
\left\|z_{n}-p\right\|^{2} \\
=\left\|T_{\delta_{n}}^{(\Theta, \varphi)}\left(x_{n}-\delta_{n} F x_{n}\right)-T_{\delta_{n}}^{(\Theta, \varphi)}\left(p-\delta_{n} F p\right)\right\|^{2} \\
\leq \leq\left\langle\left(x_{n}-\delta_{n} F x_{n}\right)-\left(p-\delta_{n} F p\right), z_{n}-p\right\rangle \\
=\frac{1}{2}\left[\left\|\left(x_{n}-\delta_{n} F x_{n}\right)-\left(p-\delta_{n} F p\right)\right\|^{2}+\left\|z_{n}-p\right\|^{2}\right. \\
\left.\quad-\left\|\left(x_{n}-\delta_{n} F x_{n}\right)-\left(p-\delta_{n} F p\right)-\left(z_{n}-p\right)\right\|^{2}\right] \\
\leq \frac{1}{2}\left[\left\|x_{n}-p\right\|^{2}+\left\|z_{n}-p\right\|^{2}-\left\|x_{n}-z_{n}-\delta_{n}\left(F x_{n}-F p\right)\right\|^{2}\right] \\
=\frac{1}{2}\left[\left\|x_{n}-p\right\|^{2}+\left\|z_{n}-p\right\|^{2}-\left\|x_{n}-z_{n}\right\|^{2}+2 \delta_{n}\left\langle F x_{n}-F p, x_{n}-z_{n}\right\rangle\right. \\
\left.\quad-\delta_{n}^{2}\left\|F x_{n}-F p\right\|^{2}\right],
\end{aligned}
$$

which implies that

$$
\left\|z_{n}-p\right\|^{2} \leq\left\|x_{n}-p\right\|^{2}-\left\|x_{n}-z_{n}\right\|^{2}+2 \delta_{n}\left\|F x_{n}-F p\right\|\left\|x_{n}-z_{n}\right\| .
$$


From (3.1), (3.4) and (3.17), we have

$$
\begin{aligned}
\left\|x_{n+1}-p\right\|^{2} & \left\|\alpha_{n}\left(\gamma f\left(W_{n} x_{n}\right)-A p\right)+\left(I-\alpha_{n} A\right)\left(W_{n} y_{n}-p\right)\right\|^{2} \\
\leq & \alpha_{n}\left\|\gamma f\left(W_{n} x_{n}\right)-A p\right\|^{2}+\left(1-\alpha_{n} \bar{\gamma}\right)\left\|y_{n}-p\right\|^{2} \\
& +2 \alpha_{n}\left\|\gamma f\left(W_{n} x_{n}\right)-A p\right\|\left\|y_{n}-p\right\| \\
\leq & \alpha_{n}\left\|\gamma f\left(W_{n} x_{n}\right)-A p\right\|^{2}+\left(1-\alpha_{n} \bar{\gamma}\right)\left\|z_{n}-p\right\|^{2} \\
& +2 \alpha_{n}\left\|\gamma f\left(W_{n} x_{n}\right)-A p\right\|\left\|y_{n}-p\right\| \\
\leq & \alpha_{n}\left\|\gamma f\left(W_{n} x_{n}\right)-A p\right\|^{2}+\left(1-\alpha_{n} \bar{\gamma}\right)\left[\left\|x_{n}-p\right\|^{2}-\left\|x_{n}-z_{n}\right\|^{2}\right. \\
& \left.+2 \delta_{n}\left\|F x_{n}-F p\right\|\left\|x_{n}-z_{n}\right\|\right]+2 \alpha_{n}\left\|\gamma f\left(W_{n} x_{n}\right)-A p\right\|\left\|y_{n}-p\right\| .
\end{aligned}
$$

It follows that

$$
\begin{aligned}
\left(1-\alpha_{n} \bar{\gamma}\right)\left\|x_{n}-z_{n}\right\|^{2} & \\
\leq & \alpha_{n}\left\|\gamma f\left(W_{n} x_{n}\right)-A p\right\|^{2}+\left\|x_{n}-p\right\|^{2}-\left\|x_{n+1}-p\right\|^{2} \\
& +2\left(1-\alpha_{n} \bar{\gamma}\right) \delta_{n}\left\|F x_{n}-F p\right\|\left\|x_{n}-z_{n}\right\|+2 \alpha_{n}\left\|\gamma f\left(W_{n} x_{n}\right)-A p\right\|\left\|y_{n}-p\right\| \\
\leq & \alpha_{n}\left\|\gamma f\left(W_{n} x_{n}\right)-A p\right\|^{2}+\left(\left\|x_{n}-p\right\|+\left\|x_{n+1}-p\right\|\right)\left\|x_{n}-x_{n+1}\right\| \\
& +2\left(1-\alpha_{n} \bar{\gamma}\right) \delta_{n}\left\|F x_{n}-F p\right\|\left\|x_{n}-z_{n}\right\|+2 \alpha_{n}\left\|\gamma f\left(W_{n} x_{n}\right)-A p\right\|\left\|y_{n}-p\right\| .
\end{aligned}
$$

Since $\left\|F x_{n}-F p\right\| \rightarrow 0$ as $n \rightarrow \infty$, we obtain

$$
\lim _{n \rightarrow \infty}\left\|x_{n}-z_{n}\right\|=0
$$

Thus, from (3.15), (3.16) and (3.18), we obtain that

$$
\begin{aligned}
\lim _{n \rightarrow \infty}\left\|z_{n}-y_{n}\right\| & =\lim _{n \rightarrow \infty}\left\|\left(z_{n}-u_{n}\right)-(p-u)+\left(u_{n}-y_{n}\right)+(p-u)\right\| \\
& \leq \lim _{n \rightarrow \infty}\left\|z_{n}-u_{n}-(p-u)\right\|+\lim _{n \rightarrow \infty}\left\|u_{n}-y_{n}+(p-u)\right\| \\
& =0
\end{aligned}
$$

and

$$
\begin{aligned}
\lim _{n \rightarrow \infty}\left\|x_{n}-y_{n}\right\| & \leq \lim _{n \rightarrow \infty}\left\|x_{n}-z_{n}\right\|+\lim _{n \rightarrow \infty}\left\|z_{n}-y_{n}\right\| \\
& =0 .
\end{aligned}
$$

Step 6. $\lim _{n \rightarrow \infty}\left\|y_{n}-W_{n} y_{n}\right\|=0$.

Indeed, observe that

$$
\begin{aligned}
\left\|x_{n}-W_{n} y_{n}\right\| & \leq\left\|x_{n}-x_{n+1}\right\|+\left\|x_{n+1}-W_{n} y_{n}\right\| \\
& =\left\|x_{n}-x_{n+1}\right\|+\left\|\alpha_{n} \gamma f\left(W_{n} x_{n}\right)+\left(I-\alpha_{n} A\right) W_{n} y_{n}-W_{n} y_{n}\right\| \\
& \leq\left\|x_{n}-x_{n+1}\right\|+\alpha_{n}\left[\gamma\left\|f\left(W_{n} x_{n}\right)\right\|+\|A\|\left\|W_{n} y_{n}\right\|\right] .
\end{aligned}
$$


From Step 3 and $\alpha_{n} \rightarrow 0$ as $n \rightarrow \infty$, we have $\lim _{n \rightarrow \infty}\left\|x_{n}-W_{n} y_{n}\right\|=0$. Consequently,

$$
\begin{aligned}
\lim _{n \rightarrow \infty}\left\|y_{n}-W_{n} y_{n}\right\| & \leq \lim _{n \rightarrow \infty}\left(\left\|y_{n}-x_{n}\right\|+\left\|x_{n}-W_{n} y_{n}\right\|\right) \\
& =0 .
\end{aligned}
$$

Step 7. $\lim \sup _{n \rightarrow \infty}\left\langle\gamma f\left(x^{*}\right)-A x^{*}, x_{n}-x^{*}\right\rangle \leq 0$, where $x^{*}=P_{\mathfrak{F}}(\gamma f+(I-A))\left(x^{*}\right)$.

Indeed, take a subsequence $\left\{x_{n_{i}}\right\}$ of $\left\{x_{n}\right\}$ such that

$$
\limsup _{n \rightarrow \infty}\left\langle\gamma f\left(x^{*}\right)-A x^{*}, x_{n}-x^{*}\right\rangle=\lim _{i \rightarrow \infty}\left\langle\gamma f\left(x^{*}\right)-A x^{*}, x_{n_{i}}-x^{*}\right\rangle .
$$

Correspondingly, there exists a subsequence $\left\{y_{n_{i}}\right\}$ of $\left\{y_{n}\right\}$. Since $\left\{y_{n_{i}}\right\}$ is bounded, there exists a subsequence of $y_{n_{i}}$ which converges weakly to $w$. Without loss of generality, we can assume that $y_{n_{i}} \rightarrow w$. Next we show $w \in \mathfrak{F}$. First, we prove that $w \in \Omega$. Utilizing Lemma 2.1, we have for all $x, y \in C$

$$
\begin{aligned}
\| \Gamma(x) & -\Gamma(y) \|^{2} \\
= & \| T_{\mu_{1}}^{G_{1}}\left[T_{\mu_{2}}^{G_{2}}\left(x-\mu_{2} B_{2} x\right)-\mu_{1} B_{1} T_{\mu_{2}}^{G_{2}}\left(x-\mu_{2} B_{2} x\right)\right] \\
& -T_{\mu_{1}}^{G_{1}}\left[T_{\mu_{2}}^{G_{2}}\left(y-\mu_{2} B_{2} y\right)-\mu_{1} B_{1} T_{\mu_{2}}^{G_{2}}\left(y-\mu_{2} B_{2} y\right)\right] \|^{2} \\
\leq & \| T_{\mu_{2}}^{G_{2}}\left(x-\mu_{2} B_{2} x\right)-T_{\mu_{2}}^{G_{2}}\left(y-\mu_{2} B_{2} y\right) \\
& -\mu_{1}\left[B_{1} T_{\mu_{2}}^{G_{2}}\left(x-\mu_{2} B_{2} x\right)-B_{1} T_{\mu_{2}}^{G_{2}}\left(y-\mu_{2} B_{2} y\right)\right] \|^{2} \\
\leq & \left\|T_{\mu_{2}}^{G_{2}}\left(x-\mu_{2} B_{2} x\right)-T_{\mu_{2}}^{G_{2}}\left(y-\mu_{2} B_{2} y\right)\right\|^{2} \\
& +\mu_{1}\left(\mu_{1}-2 \beta_{1}\right)\left\|B_{1} T_{\mu_{2}}^{G_{2}}\left(x-\mu_{2} B_{2} x\right)-B_{1} T_{\mu_{2}}^{G_{2}}\left(y-\mu_{2} B_{2} y\right)\right\|^{2} \\
\leq & \left\|T_{\mu_{2}}^{G_{2}}\left(x-\mu_{2} B_{2} x\right)-T_{\mu_{2}}^{G_{2}}\left(y-\mu_{2} B_{2} y\right)\right\|^{2} \\
\leq & \left\|x-y-\mu_{2}\left(B_{2} x-B_{2} y\right)\right\|^{2} \\
\leq & \|x-y\|^{2}+\mu_{2}\left(\mu_{2}-2 \beta_{2}\right)\left\|B_{2} x-B_{2} y\right\|^{2} \\
\leq & \|x-y\|^{2} .
\end{aligned}
$$

This shows that $\Gamma: C \rightarrow C$ is nonexpansive. Note that

$$
\begin{aligned}
\left\|y_{n}-\Gamma\left(y_{n}\right)\right\| & =\left\|\Gamma\left(z_{n}\right)-\Gamma\left(y_{n}\right)\right\| \\
& \leq\left\|z_{n}-y_{n}\right\| \\
& \rightarrow 0 \quad \text { as } n \rightarrow \infty .
\end{aligned}
$$

According to Lemma 2.2 and Lemma 2.3, we obtain $w \in \Omega$.

Next, let us show that $w \in$ GMEP. From $z_{n}=T_{\delta_{n}}^{(\Theta, \varphi)}\left(x_{n}-\delta_{n} F x_{n}\right)$, we obtain

$$
\Theta\left(z_{n}, y\right)+\varphi(y)-\varphi\left(z_{n}\right)+\frac{1}{\delta_{n}}\left\langle y-z_{n}, z_{n}-\left(x_{n}-\delta_{n} F x_{n}\right)\right) \geq 0, \quad \forall y \in C .
$$

It follows from (H2) that

$$
\varphi(y)-\varphi\left(z_{n}\right)+\left\langle y-z_{n}, F x_{n}\right\rangle+\frac{1}{\delta_{n}}\left\langle y-z_{n}, z_{n}-x_{n}\right\rangle \geq \Theta\left(y, z_{n}\right), \quad \forall y \in C .
$$


Replacing $n$ by $n_{i}$, we have

$$
\varphi(y)-\varphi\left(z_{n_{i}}\right)+\left\langle y-z_{n_{i}}, F x_{n_{i}}\right\rangle+\left\langle y-z_{n_{i}}, \frac{z_{n_{i}}-x_{n_{i}}}{\delta_{n_{i}}}\right\rangle \geq \Theta\left(y, z_{n_{i}}\right), \quad \forall y \in C .
$$

Let $z_{t}=t y+(1-t) w$ for all $t \in[0,1]$ and $y \in C$. Then we have $z_{t} \in C$. It follows from (3.19) that

$$
\begin{aligned}
\left\langle z_{t}-z_{n_{i}}, F z_{t}\right\rangle \geq & \left\langle z_{t}-z_{n_{i}}, F z_{t}\right\rangle-\varphi\left(z_{t}\right)+\varphi\left(z_{n_{i}}\right)-\left\langle z_{t}-z_{n_{i}}, F x_{n_{i}}\right\rangle \\
& -\left\langle z_{t}-z_{n_{i}}, \frac{z_{n_{i}}-x_{n_{i}}}{\delta_{n_{i}}}\right\rangle+\Theta\left(z_{t}, z_{n_{i}}\right) \\
= & \left\langle z_{t}-z_{n_{i}}, F z_{t}-F z_{n_{i}}\right\rangle+\left\langle z_{t}-z_{n_{i}}, F z_{n_{i}}-F x_{n_{i}}\right\rangle \\
& -\varphi\left(z_{t}\right)+\varphi\left(z_{n_{i}}\right)-\left\langle z_{t}-z_{n_{i}}, \frac{z_{n_{i}}-x_{n_{i}}}{\delta_{n_{i}}}\right\rangle+\Theta\left(z_{t}, z_{n_{i}}\right) .
\end{aligned}
$$

Since $\left\|z_{n_{i}}-x_{n_{i}}\right\| \rightarrow 0$, we have $\left\|F z_{n_{i}}-F x_{n_{i}}\right\| \rightarrow 0$. From the monotonicity of $F$, we have

$$
\left\langle F z_{t}-F z_{n_{i}}, z_{t}-z_{n_{i}}\right\rangle \geq 0
$$

From (H4), the weakly lower semicontinuity of $\varphi, \frac{z_{n_{i}}-x_{n_{i}}}{\delta_{n_{i}}} \rightarrow 0$ and $z_{n_{i}} \rightarrow w$, we have

$$
\left\langle z_{t}-w, F z_{t}\right\rangle \geq-\varphi\left(z_{t}\right)+\varphi(w)+\Theta\left(z_{t}, w\right)
$$

as $i \rightarrow \infty$. By (H1), (H4) and (3.20), we obtain

$$
\begin{aligned}
0 & =\Theta\left(z_{t}, z_{t}\right)+\varphi\left(z_{t}\right)-\varphi\left(z_{t}\right) \\
& =\Theta\left(z_{t}, t y+(1-t) w\right)+\varphi(t y+(1-t) w)-\varphi\left(z_{t}\right) \\
& \leq t \Theta\left(z_{t}, y\right)+(1-t) \Theta\left(z_{t}, w\right)+t \varphi(y)+(1-t) \varphi(w)-\varphi\left(z_{t}\right) \\
& =t\left[\Theta\left(z_{t}, y\right)+\varphi(y)-\varphi\left(z_{t}\right)\right]+(1-t)\left[\Theta\left(z_{t}, w\right)+\varphi(w)-\varphi\left(z_{t}\right)\right] \\
& \leq t\left[\Theta\left(z_{t}, y\right)+\varphi(y)-\varphi\left(z_{t}\right)\right]+(1-t)\left\langle z_{t}-w, F z_{t}\right\rangle \\
& =t\left[\Theta\left(z_{t}, y\right)+\varphi(y)-\varphi\left(z_{t}\right)\right]+(1-t) t\left\langle y-w, F z_{t}\right\rangle .
\end{aligned}
$$

Hence we obtain

$$
0 \leq \Theta\left(z_{t}, y\right)+\varphi(y)-\varphi\left(z_{t}\right)+(1-t)\left\langle y-w, F z_{t}\right\rangle .
$$

Putting $t \rightarrow 0$, we have

$$
0 \leq \Theta(w, y)+\varphi(y)-\varphi(w)+\langle y-w, F w\rangle, \quad \forall y \in C .
$$

This implies that $w \in$ GMEP.

Since Hilbert spaces satisfy Opial's condition, it follows from Step 5 that

$$
\begin{aligned}
\liminf _{i \rightarrow \infty}\left\|y_{n_{i}}-w\right\| & <\liminf _{i \rightarrow \infty}\left\|y_{n_{i}}-W_{n} w\right\| \\
& \leq \liminf _{i \rightarrow \infty}\left[\left\|y_{n_{i}}-W_{n} y_{n_{i}}\right\|+\left\|W_{n} y_{n_{i}}-W_{n} w\right\|\right]
\end{aligned}
$$




$$
\begin{aligned}
& \leq \limsup _{i \rightarrow \infty}\left\|y_{n_{i}}-W_{n} y_{n_{i}}\right\|+\liminf _{i \rightarrow \infty}\left\|W_{n} y_{n_{i}}-W_{n} w\right\| \\
& =\liminf _{i \rightarrow \infty}\left\|W_{n} y_{n_{i}}-W_{n} w\right\| \\
& \leq \liminf _{i \rightarrow \infty}\left\|y_{n_{i}}-w\right\|,
\end{aligned}
$$

which derives a contraction. This implies that $w \in F\left(W_{n}\right)$. It follows from $F\left(W_{n}\right)=$ $\bigcap_{i=1}^{N} F\left(T_{i}\right)$ that $w \in \bigcap_{i=1}^{N} F\left(T_{i}\right)$.

Since $x^{*}=P_{\mathfrak{F}}(\gamma f+(I-A))\left(x^{*}\right)$, we have

$$
\begin{aligned}
\limsup _{n \rightarrow \infty}\left\langle\gamma f\left(x^{*}\right)-A x^{*}, x_{n}-x^{*}\right\rangle & =\lim _{n \rightarrow \infty}\left\langle\gamma f\left(x^{*}\right)-A x^{*}, x_{n_{i}}-x^{*}\right\rangle \\
& =\left\langle\gamma f\left(x^{*}\right)-A x^{*}, w-x^{*}\right\rangle \\
& \leq 0 .
\end{aligned}
$$

Step 8. $x_{n} \rightarrow x^{*}$ as $n \rightarrow \infty$.

Indeed, from Lemma 2.6 and (3.4), we have

$$
\begin{aligned}
&\left\|x_{n+1}-x^{*}\right\|^{2} \\
&=\left\|\alpha_{n} \gamma f\left(W_{n} x_{n}\right)+\left(I-\alpha_{n} A\right) W_{n} y_{n}-x^{*}\right\|^{2} \\
&=\left\|\left(I-\alpha_{n} A\right)\left(W_{n} y_{n}-x^{*}\right)+\alpha_{n}\left(\gamma f\left(W_{n} x_{n}\right)-A x^{*}\right)\right\|^{2} \\
& \leq\left\|\left(I-\alpha_{n} A\right)\left(W_{n} y_{n}-x^{*}\right)\right\|^{2}+2 \alpha_{n}\left\langle\gamma f\left(W_{n} x_{n}\right)-A x^{*}, x_{n+1}-x^{*}\right\rangle \\
& \leq\left(1-\alpha_{n} \bar{\gamma}\right)^{2}\left\|y_{n}-x^{*}\right\|^{2}+2 \alpha_{n}\left\langle\gamma f\left(W_{n} x_{n}\right)-A x^{*}, x_{n+1}-x^{*}\right\rangle \\
& \leq\left(1-\alpha_{n} \bar{\gamma}\right)^{2}\left\|x_{n}-x^{*}\right\|^{2}+2 \alpha_{n} \gamma\left\langle f\left(W_{n} x_{n}\right)-f\left(x^{*}\right), x_{n+1}-x^{*}\right\rangle \\
&+2 \alpha_{n}\left\langle\gamma f\left(x^{*}\right)-A x^{*}, x_{n+1}-x^{*}\right\rangle \\
& \leq\left(1-\alpha_{n} \bar{\gamma}\right)^{2}\left\|x_{n}-x^{*}\right\|^{2}+2 \alpha_{n} \gamma \alpha\left\|x_{n}-x^{*}\right\|\left\|x_{n+1}-x^{*}\right\| \\
&+2 \alpha_{n}\left\langle\gamma f\left(x^{*}\right)-A x^{*}, x_{n+1}-x^{*}\right\rangle \\
& \leq\left(1-\alpha_{n} \bar{\gamma}\right)^{2}\left\|x_{n}-x^{*}\right\|^{2}+\alpha_{n} \gamma \alpha\left(\left\|x_{n}-x^{*}\right\|^{2}+\left\|x_{n+1}-x^{*}\right\|^{2}\right) \\
&+2 \alpha_{n}\left\langle\gamma f\left(x^{*}\right)-A x^{*}, x_{n+1}-x^{*}\right\rangle,
\end{aligned}
$$

which implies that

$$
\begin{aligned}
& \left\|x_{n+1}-x^{*}\right\|^{2} \\
& \leq \quad \frac{\left(1-\alpha_{n} \bar{\gamma}\right)^{2}+\alpha_{n} \gamma \alpha}{1-\alpha_{n} \gamma \alpha}\left\|x_{n}-x^{*}\right\|^{2}+\frac{2 \alpha_{n}}{1-\alpha_{n} \gamma \alpha}\left\langle\gamma f\left(x^{*}\right)-A x^{*}, x_{n+1}-x^{*}\right\rangle \\
& =\left(1-\frac{2 \alpha_{n}(\bar{\gamma}-\alpha \gamma)}{1-\alpha_{n} \gamma \alpha}\right)\left\|x_{n}-x^{* *}\right\|^{2} \\
& \quad+\frac{2 \alpha_{n}(\bar{\gamma}-\alpha \gamma)}{1-\alpha_{n} \gamma \alpha}\left[\frac{\alpha_{n} \bar{\gamma}^{2}}{2(\bar{\gamma}-\alpha \gamma)}\left\|x_{n}-x^{*}\right\|^{2}+\frac{1}{\bar{\gamma}-\alpha \gamma}\left\langle\gamma f\left(x^{*}\right)-A x^{*}, x_{n+1}-x^{*}\right\rangle\right] .
\end{aligned}
$$


Put

$$
\gamma_{n}=\frac{2 \alpha_{n}(\bar{\gamma}-\alpha \gamma)}{1-\alpha_{n} \gamma \alpha}
$$

and

$$
\xi_{n}=\frac{2 \alpha_{n}(\bar{\gamma}-\alpha \gamma)}{1-\alpha_{n} \gamma \alpha}\left[\frac{\alpha_{n} \bar{\gamma}^{2}}{2(\bar{\gamma}-\alpha \gamma)}\left\|x_{n}-x^{*}\right\|^{2}+\frac{1}{\bar{\gamma}-\alpha \gamma}\left\langle\gamma f\left(x^{*}\right)-A x^{*}, x_{n+1}-x^{*}\right\rangle\right] .
$$

Then we can write the last inequality as

$$
a_{n+1} \leq\left(1-\gamma_{n}\right) a_{n}+\xi_{n}
$$

It follows from condition (i) and Step 6 that

$$
\sum_{n=1}^{\infty} \gamma_{n}=+\infty
$$

and

$$
\begin{aligned}
\limsup _{n \rightarrow \infty} \frac{\xi_{n}}{\gamma_{n}}= & \limsup _{n \rightarrow \infty}\left\{\frac{\alpha_{n} \bar{\gamma}^{2}}{2(\bar{\gamma}-\alpha \gamma)}\left\|x_{n}-x^{*}\right\|^{2}\right. \\
& \left.+\frac{1}{\bar{\gamma}-\alpha \gamma}\left\langle\gamma f\left(x^{*}\right)-A x^{*}, x_{n+1}-x^{*}\right\rangle\right\} \\
\leq & 0 .
\end{aligned}
$$

Hence, applying Lemma 2.4, we immediately obtain that $x_{n} \rightarrow x^{*}$ as $n \rightarrow \infty$. This completes the proof.

As corollaries of Theorem 3.1, we have the following results.

Corollary 3.1 Let $C$ be a nonempty closed convex subset of a real Hilbert space H. Let $\Theta, G_{1}, G_{2}: C \times C \rightarrow \mathbb{R}$ be three bifunctions which satisfy assumptions (H1)-(H4) and $\varphi: C \rightarrow \mathbb{R}$ be a lower semicontinuous and convex function satisfying (A1) or (A2). Let the mappings $B_{1}, B_{2}: C \rightarrow H$ be $\beta_{1}$-inverse strongly monotone and $\beta_{2}$-inverse strongly monotone, respectively. Let $T_{1}, T_{2}, \ldots, T_{N}$ be a finite family of nonexpansive mappings of $C$ into $H$ such that $\mathfrak{F}=\bigcap_{i=1}^{N} F\left(T_{i}\right) \cap \mathrm{MEP} \cap \Omega \neq \phi$. Let $f$ be a contraction of $H$ into itself with a constant $\alpha(0<\alpha<1)$ and let $A$ be a strongly positive linear bounded operator with a coeffcient $\bar{\gamma}>0$ such that $\|A\| \leq 1$. Assume that $0<\gamma<\frac{\bar{\gamma}}{\alpha}$. Let $x_{1} \in C$ and let $\left\{x_{n}\right\}$ be a sequence defined by

$$
\left\{\begin{array}{l}
\Theta\left(z_{n}, y\right)+\varphi(y)-\varphi\left(z_{n}\right)+\frac{1}{\delta_{n}}\left\langle y-z_{n}, z_{n}-x_{n}\right\rangle \geq 0, \quad \forall y \in C, \\
y_{n}=T_{\mu_{1}}^{G_{1}}\left[T_{\mu_{2}}^{G_{2}}\left(z_{n}-\mu_{2} B_{2} z_{n}\right)-\mu_{1} B_{1} T_{\mu_{2}}^{G_{2}}\left(z_{n}-\mu_{2} B_{2} z_{n}\right)\right], \\
x_{n+1}=\alpha_{n} \gamma f\left(W_{n} x_{n}\right)+\left(1-\alpha_{n} A\right) W_{n} y_{n}, \quad n \geq 1,
\end{array}\right.
$$

where $\alpha_{n} \in[0,1], \mu_{1} \in\left(0,2 \beta_{1}\right), \mu_{2} \in\left(0,2 \beta_{2}\right)$ and $\left\{\delta_{n}\right\} \subset(0, \infty)$ satisfy the following conditions: 
(i) $\lim _{n \rightarrow \infty} \alpha_{n}=0, \sum_{n=1}^{\infty} \alpha_{n}=\infty$ and $\sum_{n=1}^{\infty}\left|\alpha_{n+1}-\alpha_{n}\right|<\infty$;

(ii) $\lim _{n \rightarrow \infty} \lambda_{n, i}=0, \sum_{n=1}^{\infty}\left|\lambda_{n, i}-\lambda_{n-1, i}\right|<\infty$ for all $i=1,2, \ldots, N$,

(iii) $0<\liminf _{n \rightarrow \infty} \delta_{n} \leq \lim \sup _{n \rightarrow \infty} \delta_{n}<\infty$ and $\sum_{n=1}^{N}\left|\delta_{n+1}-\delta_{n}\right|<\infty$.

Then $\left\{x_{n}\right\}$ converges strongly to $x^{*}=P_{\bigcap_{i=1}^{N} F\left(T_{i}\right) \cap \mathrm{MEP} \cap \Omega}(\bar{\gamma} f+(I-A))\left(x^{*}\right)$ and $\left(x^{*}, y^{*}\right)$ is a solution of problem (1.4), where $y^{\prime \prime}=T_{\mu_{2}}^{G_{2}}\left(x^{* \prime}-\mu_{2} B_{2} x^{*}\right)$, which solves the following variational inequality:

$$
\left\langle(A-\gamma f) x^{*}, x-x^{*}\right\rangle \geq 0, \quad \forall x \in \bigcap_{i=1}^{N} F\left(T_{i}\right) \cap \operatorname{MEP} \cap \Omega .
$$

Proof In Theorem 3.1, for all $n \geq 0, z_{n}=T_{\delta n}^{(\Theta, \varphi)}\left(x_{n}-\delta_{n} F x_{n}\right)$ is equivalent to

$$
\Theta\left(z_{n}, y\right)+\varphi(y)-\varphi\left(z_{n}\right)+\left\langle F x_{n}, y-z_{n}\right\rangle+\frac{1}{\delta_{n}}\left\langle y-z_{n}, z_{n}-x_{n}\right\rangle \geq 0, \quad \forall y \in C .
$$

Putting $F \equiv 0$, we obtain

$$
\Theta\left(z_{n}, y\right)+\varphi(y)-\varphi\left(z_{n}\right)+\frac{1}{\delta_{n}}\left\langle y-z_{n}, z_{n}-x_{n}\right\rangle \geq 0, \quad \forall y \in C .
$$

Corollary 3.2 Let $C$ be a nonempty closed convex subset of a real Hilbert space $H$ and let $G_{1}, G_{2}: C \times C \rightarrow \mathbb{R}$ be two bifunctions with satisfy assumptions (H1)-(H4). Let the mappings $F, B_{1}, B_{2}: C \rightarrow H$ be $\zeta$-inverse strongly monotone, $\beta_{1}$-inverse strongly monotone and $\beta_{2}$-inverse strongly monotone, respectively. Let $T_{1}, T_{2}, \ldots, T_{N}$ be a finite family of nonexpansive mappings of $C$ into $H$ such that $\bigcap_{i=1}^{N} F\left(T_{i}\right) \cap \operatorname{VI}(A, C) \cap \Omega \neq \phi$. Let $f$ be a contraction of $H$ into itself with a constant $\alpha(0<\alpha<1)$ and let $A$ be a strongly positive linear bounded operator with a coefficient $\bar{\gamma}>0$ such that $\|A\| \leq 1$. Assume that $0<\gamma<\frac{\bar{\gamma}}{\alpha}$. Let $x_{1} \in C$ and let $\left\{x_{n}\right\}$ be a sequence defined by

$$
\left\{\begin{array}{l}
z_{n}=P_{C}\left(x_{n}-\delta F x_{n}\right), \\
y_{n}=T_{\mu_{1}}^{G_{1}}\left[T_{\mu_{2}}^{G_{2}}\left(z_{n}-\mu_{2} B_{2} z_{n}\right)-\mu_{1} B_{1} T_{\mu_{2}}^{G_{2}}\left(z_{n}-\mu_{2} B_{2} z_{n}\right)\right], \\
x_{n+1}=\alpha_{n} \gamma f\left(W_{n} x_{n}\right)+\left(1-\alpha_{n} A\right) W_{n} y_{n}, \quad n \geq 1,
\end{array}\right.
$$

where $\alpha_{n} \in[0,1], \mu_{1} \in\left(0,2 \beta_{1}\right), \mu_{2} \in\left(0,2 \beta_{2}\right)$ and $\left\{\delta_{n}\right\} \subset[0,2 \zeta]$ satisfy the following conditions:

(i) $\lim _{n \rightarrow \infty} \alpha_{n}=0, \sum_{n=1}^{\infty} \alpha_{n}=0$ and $\sum_{n=1}^{\infty}\left|\alpha_{n+1}-\alpha_{n}\right|<\infty$;

(ii) $\lim _{n \rightarrow \infty} \lambda_{n, i}=0$ and $\sum_{n=1}^{\infty}\left|\lambda_{n, i}-\lambda_{n-1, i}\right|<\infty$ for all $i=1,2, \ldots, N$;

(iii) $\liminf _{n \rightarrow \infty} \delta_{n}>0$ and $\sum_{n=1}^{\infty}\left|\delta_{n+1}-\delta_{n}\right|<\infty$.

Then $\left\{x_{n}\right\}$ converges strongly to $x^{*}=P_{\bigcap_{i=1}^{N} F\left(T_{i}\right) \cap \mathrm{VI}(A, C) \cap \Omega}(\gamma f+(I-A))\left(x^{*}\right)$ and $\left(x^{*}, y^{*}\right)$ is a solution of problem (1.4), where $y^{*}=T_{\mu_{2}}^{G_{2}}\left(x^{*}-\mu_{2} B_{2} x^{*}\right)$, which solves the following variational inequality:

$$
\left\langle(A-\gamma f) x^{*}, x-x^{*}\right| \geq 0, \quad \forall x \in \bigcap_{i=1}^{N} F\left(T_{i}\right) \cap \operatorname{VI}(A, C) \cap \Omega .
$$

Proof Put $\Theta=0$ and $\varphi=0$ in Theorem 3.1. Then we have from (3.21) that

$$
\left\langle F x_{n}, y-z_{n}\right\rangle+\frac{1}{\delta_{n}}\left\langle y-z_{n}, z_{n}-x_{n}\right\rangle \geq 0, \quad \forall y \in C, n \geq 1 .
$$


That is,

$$
\left\langle y-z_{n}, x_{n}-\delta_{n} F x_{n}-z_{n}\right\rangle \leq 0, \quad \forall y \in C
$$

It follows that $P_{C}\left(x_{n}-\delta_{n} F x_{n}\right)=z_{n}$ for all $n \geq 1$. We can obtain the desired conclusion easily.

\section{Remark 3.1 We can see easily that Takahashi and Takahashi [18], Peng and Yao's [1] results are special cases of Theorem 3.1.}

\section{Competing interests}

The author declares that they have no competing interests.

\section{Acknowledgements}

The author is grateful to the referees for useful suggestions that improved the contents of the article.

Received: 4 June 2012 Accepted: 1 March 2013 Published: 21 March 2013

\section{References}

1. Peng, JW, Yao, JC: A new hybrid extragradient method for generalized mixed equilibrium problems, fixed point problems and variational inequality problems. Taiwan. J. Math. 12, 1401-1432 (2008)

2. Ceng, LC, Yao, JC: A hybrid iterative scheme for mixed equilibrium problems and fixed point problems. J. Comput. Appl. Math. 214, 186-201 (2008)

3. Takahashi, S, Takahashi, W: Strong convergence theorem for a generalized equilibrium problem and a nonexpansive mapping in a Hilbert space. Nonlinear Anal. 69, 1025-1033 (2008)

4. Chang, SS, Lee, HWJ, Chan, CK: A new method for solving equilibrium problem fixed point problem and variational inequality problem with application to optimization. Nonlinear Anal. 70, 3307-3319 (2009)

5. Ding, XP: Iterative algorithm of solutions for a system of generalized mixed implicity equilibrium problems in reflexive Banach spaces. Appl. Math. Comput. 218, 4953-4961 (2012)

6. Jaiboon, C, Kumam, P: A general iterative method for addressing mixed equilibrium problems and optimization problems. Nonlinear Anal. 73, 1180-1202 (2010)

7. Moudafi, A: Mixed equilibrium problems: sensitivity analysis and algorithmic aspect. Comput. Math. Appl. 44 1099-1108 (2002)

8. Qin, X, Shang, M, Su, Y: Strong convergence of a general iterative algorithm for equilibrium problems and variational inequality problems. Math. Comput. Model. 48, 1033-1046 (2008)

9. Yang, S, Li, W: Iterative solutions of a system of equilibrium problems in Hilbert spaces. Adv. Fixed Point Theory 1 , 15-26 (2011)

10. Ye, J, Huang, J: Strong convergence theorems for fixed point problems and generalized equilibrium problems of three relatively quasi-nonexpansive mappings in Banach spaces. J. Math. Comput. Sci. 1, 1-18 (2011)

11. Blum, E, Oettli, W: From optimization and variational inequalities to equilibrium problems. Math. Stud. 63, 123-145 (1994)

12. Ceng, LC, Wang, CY, Yao, JC: Strong convergence theorems by a relaxed extragradient method for a general system of variational inequalities. Math. Methods Oper. Res. 67, 375-390 (2008)

13. Verma, RU: On a new system of nonlinear variational inequalities and associated iterative algorithms. Math. Sci. Res. Hot-Line 3, 65-68 (1999)

14. Chen, JM, Zhang, LJ, Fan, TG: Viscosity approximation methods for nonexpansive mappings and monotone mappings. J. Math. Anal. Appl. 334, 1450-1461 (2007)

15. Combettes, PL, Hirstoaga, SA: Equilibrium programming in Hilbert spaces. J. Nonlinear Convex Anal. 6, 117-136 (2005)

16. liduka, $H$, Takahashi, W: Strong convergence theorems for nonexpansive mappings and inverse strongly monotone mappings. Nonlinear Anal. 61, 341-350 (2005)

17. Marino, G, Xu, HK: A general iterative method for nonexpansive mappings in Hilbert spaces. J. Math. Anal. Appl. 318 43-52 (2006)

18. Takahashi, S, Takahashi, W: Viscosity approximation methods for equilibrium problems and fixed point problems in Hilbert spaces. J. Math. Anal. Appl. 331, 506-515 (2007)

19. Wittmann, R: Approximation of fixed points of nonexpansive mappings. Arch. Math. 58, 486-491 (1992)

20. Opial, Z: Weak convergence of the sequence of successive approximations for nonexpansive mappings. Bull. Am. Math. Soc. 73, 595-597 (1967)

21. Goebel, K, Kirk, WA: Topics on Metric Fixed Point Theory. Cambridge University Press, Cambridge (1990)

22. Xu, HK: Iterative algorithms for nonlinear operators. J. Lond. Math. Soc. 66, 240-256 (2002) 